\title{
Cohomology of graph hypersurfaces associated to certain Feynman graphs
}

\author{
DZMITRY DORYN
}

\begin{abstract}
To any Feynman graph (with $2 n$ edges) we can associate a hypersurface $X \subset \mathbb{P}^{2 n-1}$. We study the cohomology of the middle degree $H^{2 n-2}(X)$ of such graph hypersurface. Bloch et al. (Commun. Math. Phys. 267, 2006) have computed this cohomology for the first series of examples, the wheel with spokes $W S_{n}, n \geq 3$. Using the same technique, we introduce the generalized zigzag graphs and prove that $W_{5}\left(H^{2 n-2}(X)\right)=\mathbb{Q}(-2)$ for all of them (with $W_{*}$ the weight filtration). We also can compute $\# X\left(\mathbb{F}_{q}\right) \equiv 1+q+$ $2 q^{2} \bmod q^{3}$ for the number of rational points of such hypersurface. At the end, we study the behavior of graph hypersurfaces under the gluing of graphs.
\end{abstract}

\section{Introduction}

In the last decades, the renormalization group functions, i.e. beta-function and anomalous dimensions of field operations, were computed to high orders in the perturbative expansion in terms of Feynman graphs. The greatest computational challenge is provided by the renormalization-scheme independent contributions to such computations. They are provided by Feynman graphs without divergent subgraphs - primitively log divergent graphs which hence evaluate to a single period as a coefficient of their scaling behavior.

In [1] Broadhurst and Kreimer have done these computations up to graphs with Betti number seven ("7 loops," in physics parlance) in the mid1990s.

A particular simple class of graphs are the wheel with $n$ spokes graphs $\mathrm{WS}_{n}$, which were considered as a warm-up in [1], and are proven to deliver periods proportional to $\zeta(2 n-3)$ at $n$ loops.

It is those graphs which were investigated by Bloch et al. in [2] and finally showed that there is a Hodge-theoretic (or motivic, of one dares) rhyme and reason for the appearance of this period. More precisely, let $X_{n} \subset \mathbb{P}^{2 n-1}$ be 
the graph hypersurface for the graph $\mathrm{WS}_{n}$; it was proved that (as a Hodge structure)

$$
H_{\mathrm{c}}^{2 n-1}\left(\mathbb{P}^{2 n-1} \backslash X\right) \cong \mathbb{Q}(-2)
$$

and that the de Rham cohomology $H_{\mathrm{DR}}^{2 n-1}\left(\mathbb{P}^{2 n-1} \backslash X\right)$ is generated by the integrand of the graph period (2.9).

This paper is a natural continuation of this result. Our aim was to settle more general classes of graphs that can be studied with similar methods. Cranking the level of difficulty up one notch, the zigzag graphs are genuine graphs appearing in $\Phi^{4}$ theory and were numerically computed by Broadhurst and Kreimer, who then conjectured a general formula for the period of zigzag graph with the Betti number equals $n, Z Z_{n}$ being of the form $q(n) \zeta(2 n-3)$ with $q(n)$ rational.

After the new numerical results on the periods of graphs in $\Phi^{4}$ theory given by Schnetz in [3], we have no hope to control the rational coefficients and configurations of MZV in the periods in general. But we believe that the cohomological results can recognize the weight of MZV. So, if there appears the weight drop (see [4] for definition and combinatorial explanation), we can read this off from the associated mixed Hodge structure.

Here we obtain the same Hodge-theoretical result for the graph $\mathrm{ZZ}_{5}$ as in [2]. We define a big series (which contains $Z Z_{n}$ ) of graphs for which the maximal nontrivial weight piece of the Hodge structure is of Tate type: $\operatorname{gr} \underset{\max }{W} H_{\mathrm{c}}^{2 n-1}\left(\mathbb{P}^{2 n-1} \backslash X\right) \cong \mathbb{Q}(-2)$ (Theorem 4.2).

Using the same stratification of a graph hypersurface as in this proof and the technique of counting rational points using $\ell$-adic cohomology, we get the congruence $\# X\left(\mathbb{F}_{q}\right) \equiv 1+q+2 q^{2} \bmod q^{3}$. We study gluings (two vertex joins) of primitively log divergent graphs and compute $\operatorname{gr}_{\max }^{W} H_{\mathrm{c}}^{2 n-1}$ $\left(\mathbb{P}^{2 n-1} \backslash X\right)=\mathbb{Q}(-3)$ for the case of gluing of two graphs $W S_{3}$ and $W S_{n}$.

This paper is organized as follows. Section 1 contains some theorems on determinants, this is a key ingredient of our computation. The second section is a remainder of the construction of graph polynomials and periods. The cohomological tools are presented in Section 3.

We define the generalized zigzag graphs GZZ and prove that they are primitively log divergent in Section 4 . Then we present the main result that the minimal nontrivial weight piece of the mixed Hodge structure associated to the cohomology of such graph hypersurface is Tate (Theorem 4.2). We get a more concrete result for the cohomology of the middle degree of the graph $\mathrm{ZZ}_{5}$ (Theorem 4.3). Then we count the number of rational points of GZZ (Theorem 4.4). 
In Section 5 it is proved that the integrand (2.9) is nonzero in the de Rham cohomology $H_{\mathrm{DR}}^{2 n-2}\left(\mathbb{P}^{2 n-1} \backslash X\right)$ for the subset $\operatorname{GZZ}(n, 2)$ of $\mathrm{GZZ}, n \geq 2$, and generates it in the case of $\mathrm{ZZ}_{5}$.

In Section 6 we define the gluing $\Gamma \times \Gamma^{\prime}$ of two graphs $\Gamma$ and $\Gamma$ and do computation for the cohomology of the middle degree for $\mathrm{WS}_{3} \times \mathrm{WS}_{n}$.

\section{Determinants}

Theorem 1.1. Let $R$ be a commutative ring with 1. Consider a free rang $n+1 R$-module $V$. Then there is a canonical isomorphism

$$
\bigwedge^{2}\left(\bigwedge^{n} V\right)=\bigwedge^{n-1} V \otimes \operatorname{det} V
$$

Proof. Using the perfect pairings $\bigwedge^{i} V \otimes \bigwedge^{n+1-i} V \longrightarrow \bigwedge^{n+1} V=: \operatorname{det} V$ for $i=1$ and $i=2$ and denoting by $V^{\vee}$ the dual module, we compute

$$
\begin{aligned}
\bigwedge^{2}\left(\bigwedge^{n} V\right) & =\bigwedge^{2}\left(V^{\vee}\right) \otimes(\operatorname{det} V)^{2}=\bigwedge^{n-1} V \otimes(\operatorname{det} V)^{-1} \otimes(\operatorname{det} V)^{2} \\
& =\bigwedge^{n-1} V \otimes \operatorname{det} V .
\end{aligned}
$$

Let $\mathcal{M}=\left(a_{i j}\right)_{0 \leq i, j \leq n}$ be an $(n+1) \times(n+1)$-matrix with entries in $R$. The numeration of rows and columns goes 0 through $n$. Let $\mathcal{M}\left(i_{0}, \ldots, i_{k} ; j_{0}, \ldots\right.$, $j_{t}$ ) be the submatrix which we get from the matrix after removing rows $i_{0}$ to $i_{k}$ and columns $j_{0}$ to $j_{t}$. It is very convenient to denote the determinant of $\mathcal{M}$ just by $M$. We assume that the determinant of zero-dimensional matrix is 1 . For example, $M(0, n ; 0, n)=1$ for the matix in the definition above with $t=k=n=1$.

Corollary 1.1. Let $n \geq 1$. For any $(n+1) \times(n+1)$-matrix $\mathcal{M}$ and any integers $0 \leq i, j, k, t \leq n$, satisfying $i \neq k$ and $j \neq t$, we have

$$
M(i ; j) M(k ; t)-M(k ; j) M(i ; t)=M \cdot M(i, k ; j, t) .
$$

Proof. The action of $\mathcal{M}$ on $V$ induces the action on both sides of (1.1). For a basis $e_{0}, \ldots, e_{n}$ of $V$, one considers the coefficients of the elements corresponding to $e_{i}^{\vee}, e_{k}^{\vee}$ for the first copy of $\bigwedge^{n} V$ on the left, and $e_{j}^{\vee}, e_{t}^{\vee}$ for the second. The formula follows. 
For a matrix $\mathcal{M}=\left(a_{i j}\right)_{0 \leq i, j \leq n}$, we define the minors

$$
\begin{aligned}
I_{k}^{i}:= & M(0,1, \ldots, i-1, i+k, i+k+1, \ldots, n \\
& 0,1, \ldots, i-1, i+k, i+k+1, \ldots, n),
\end{aligned}
$$

where $1 \leq k \leq n+1$ and $1 \leq i \leq n$. We usually write $I_{n}$ for $I_{n}^{0}$. For example, $I_{n+1}=M, I_{n}^{1}=M(0 ; 0)$ and $I_{n}=M(n ; n)$.

Corollary 1.2. For a symmetric matrix $\mathcal{M}=\left(a_{i j}\right)_{0 \leq i, j \leq n}$ one has the following equality:

$$
I_{n} I_{n}^{1}-I_{n-1}^{1} I_{n+1}=(M(0 ; n))^{2}
$$

Take now an $(n+1) \times(n+1)$-matrix $\mathcal{M}=\left(a_{i j}\right)$ with entries in $R$ and suppose that the transpose of the last row equals the last column with elements, renumbered by single lower indices.

$$
\mathcal{M}=\left(\begin{array}{cccccc}
a_{00} & a_{01} & \vdots & a_{0 n-2} & a_{0 n-1} & a_{0} \\
a_{10} & a_{11} & \vdots & a_{1 n-2} & a_{1 n-1} & a_{1} \\
\ldots & \ldots & \ddots & \ldots & \ldots & \ldots \\
a_{n-20} & a_{n-21} & \vdots & a_{n-2 n-2} & a_{n-2 n-1} & a_{n-2} \\
a_{n-10} & a_{n-11} & \vdots & a_{n-1 n-2} & a_{n-1 n-1} & a_{n-1} \\
a_{0} & a_{1} & \vdots & a_{n-2} & a_{n-1} & a_{n}
\end{array}\right)
$$

The determinant of $\mathcal{M}$ is thought of as an element in $R\left[a_{0}, \ldots, a_{n}\right]$. It can be written as $M=I_{n+1}=a_{n} I_{n}-G_{n}$. Then $G_{n}$ is computed as

$$
G_{n}:=\sum_{0 \leq i, j \leq n-1}(-1)^{i+j} a_{i} a_{j} I_{n}(i ; j) .
$$

The entries $a_{i}$ play the role of variables while the other entries and minors are coefficients. The polynomial $G_{n} \in R\left[a_{0}, \ldots, a_{n}\right]$ is of degree 2 . We claim

Theorem 1.2. Let $I_{n-1} \not \equiv 0 \bmod I_{n}$. Then

$$
I_{n-1} G_{n} \equiv L_{n} L_{n}^{\prime} \bmod I_{n}
$$

for some $L_{n}$ and $L_{n}^{\prime}$, linear as polynomials of the "variables." 
Proof. By Corollory 1.1, we have

$$
I_{n}(i ; j) I_{n}(n-1 ; n-1) \equiv I_{n}(i ; n-1) I_{n}(n-1 ; j) \bmod I_{n}
$$

for all $1 \leq i, j \leq n-2$. We multiply $G_{n}$ by $I_{n-1}=I_{n}(n-1, n-1)$ and get

$$
\begin{aligned}
I_{n-1} G_{n}= & a_{n-1}^{2}\left(I_{n-1}\right)^{2}+\sum_{0 \leq i, j \leq n-2}(-1)^{i+j} a_{i} a_{j} I_{n-1} I_{n}(i ; j) \\
& +a_{n-1} \sum_{0 \leq i \leq n-2}(-1)^{i+n-1} a_{i} I_{n-1}\left(I_{n}(i ; n-1)+I_{n}(n-1 ; i)\right) \\
\equiv & \left(a_{n-1} I_{n-1}+\sum_{0 \leq i \leq n-2}(-1)^{i+n-1} a_{i} I_{n}(i ; n-1)\right) \\
& \times\left(a_{n-1} I_{n-1}+\sum_{0 \leq j \leq n-2}(-1)^{j+n-1} a_{j} I_{n}(n-1 ; j)\right) \bmod I_{n} .
\end{aligned}
$$

We set

$$
L_{n}=a_{n-1} I_{n-1}+\sum_{0 \leq i \leq n-2}(-1)^{i+n-1} a_{i} I_{n}(i ; n-1)
$$

and

$$
L_{n}^{\prime}=a_{n-1} I_{n-1}+\sum_{0 \leq j \leq n-2}(-1)^{j+n-1} a_{i} I_{n}(n-1 ; j) .
$$

In the next sections we deal only with symmetric matrices, and thus we make a

Corollary 1.3. Let $\mathcal{M}=\left(a_{i j}\right)$ be a symmetric $(n+1) \times(n+1)$-matrix with entries in a ring $R$ (see (1.6)). If $I_{n-1} \not \equiv 0 \bmod I_{n}$, the congruence

$$
I_{n-1} G_{n} \equiv\left(L_{n}\right)^{2} \bmod I_{n}
$$

holds, where $G_{n}$ and $L_{n}$ are given by (1.7) and (1.11), respectively.

One more fact about $G_{n}$ will be used frequently in the next sections. 
Theorem 1.3. Let $\mathcal{M}=\left(a_{i j}\right)$ be a symmetric $(n+1) \times(n+1)$-matrix with entries in a ring $R$ and assume that the quotient ring $R /\left(I_{n}\right)$ is a domain. If $I_{n-1} \equiv 0 \bmod I_{n}$, then

$$
G_{n} \equiv \sum_{0 \leq i \leq n-2} a_{i}^{2} I_{n}(i ; i)+2 \sum_{0 \leq i<j \leq n-2}(-1)^{i+j} a_{i} a_{j} I_{n}(i ; j) \bmod I_{n} .
$$

Proof. We prove that $G_{n}$ forgets the "variable" $a_{n-1}$. Since $\mathcal{M}$ is symmetric, we can rewrite $G_{n}$ (see (1.7)) as

$$
\begin{aligned}
G_{n}= & a_{n-1}^{2} I_{n-1}+\sum_{0 \leq i \leq n-2}(-1)^{i+n-1} a_{i} a_{n-1}\left(I_{n}(i ; n-1)+I_{n}(n-1 ; i)\right) \\
& +\sum_{0 \leq i \leq j \leq n-2}(-1)^{i+j} a_{i} a_{j} I_{n}(i ; j) \\
= & a_{n-1}^{2} I_{n-1}+2 \sum_{0 \leq i \leq n-2}(-1)^{i+n-1} a_{i} a_{n-1} I_{n}(i ; n-1) \\
& +\sum_{0 \leq i \leq n-2} a_{i}^{2} I_{n}(i ; i)+2 \sum_{0 \leq i<j \leq n-2}(-1)^{i+j} a_{i} a_{j} I_{n}(i ; j) .
\end{aligned}
$$

Corollory 1.1 implies $\left(I_{n}(i ; n-1)\right)^{2} \equiv I_{n}(i ; i) I_{n}(n-1 ; n-1) \bmod I_{n}$ for all $0 \leq i \leq n-2$. Since $R /\left(I_{n}\right)$ is a domain and $I_{n-1}=I_{n}(n-1 ; n-1) \equiv 0$ $\bmod I_{n}$, we get $I_{n}(i ; n-1) \equiv 0 \bmod I_{n}$. Hence, (1.15) implies the congruence

$$
G_{n} \equiv \sum_{0 \leq i \leq n-2} a_{i}^{2} I_{n}(i ; i)+2 \sum_{0 \leq i<j \leq n-2}(-1)^{i+j} a_{i} a_{j} I_{n}(i ; j) \bmod I_{n} .
$$

Remark 1.1. In our computations we will apply Corollary 1.3 and Theorem 1.2 only for the case where $R$ is a polynomial ring over an algebraically closed field of characteristic zero. Moreover, entries of matrices will be only linear polynomials in $R$.

\section{Graph polynomials}

Let $\Gamma$ be a finite graph with edges $E$ and vertices $V$. We choose an orientation of edges. For a given vertex $v$ and a given edge $e$, we define $\operatorname{sign}(e, v)$ to be -1 if $e$ enters $v$ and +1 if $e$ exits $v$. Denote by $\mathbb{Z}[E]$ (resp. $\mathbb{Z}[V]$ ) the free $\mathbb{Z}$-module generated by the elements of $E$ (resp. $V$ ). Consider the homology 
sequence

$$
0 \longrightarrow H_{1}(\Gamma, \mathbb{Z}) \stackrel{\iota}{\longrightarrow} \mathbb{Z}[E] \stackrel{\partial}{\longrightarrow} \mathbb{Z}[V] \longrightarrow H_{0}(\Gamma, \mathbb{Z}) \longrightarrow 0
$$

where the $\mathbb{Z}$-linear map $\partial$ is defined by $\partial(e)=\sum_{v \in V} \operatorname{sign}(v, e)$. The elements $e^{\vee}$ of a dual basis of $\mathbb{Z}[E]$ define linear forms $e^{\vee} \circ \iota$ on $H=H_{1}(\Gamma, \mathbb{Z})$. We view the squares of these functions $\left(e^{\vee} \circ \iota\right)^{2}: H \rightarrow \mathbb{Z}$ as rank 1 quadratic forms. For a fixed basis of $H$ we can associate a rank 1 symmetric matrix $\mathcal{M}_{e}$ to each such form.

Definition 2.1. We define the graph polynomial of $\Gamma$

$$
\Psi_{\Gamma}:=\operatorname{det}\left(\sum_{e \in E} A_{e} \mathcal{M}_{e}\right)
$$

in some variables $A_{e}$.

The polynomial $\Psi$ is homogeneous of degree rank $H$. A change of the basis of $H$ only changes $\Psi_{\Gamma}$ by +1 or -1 .

Definition 2.2. The Betti number of a graph $\Gamma$ is defined to be $h_{1}(\Gamma):=$ $\operatorname{rank} H_{1}(\Gamma, \mathbb{Z})$.

The definition of $\Psi_{\Gamma}$ agrees with the other well-known definition (see $[2$, Proposition 2.2]):

Proposition 2.1. One has

$$
\Psi_{\Gamma}(A)=\sum_{T \text { span tr. }} \prod_{e \notin T} A_{e}
$$

Corollary 2.1. The coefficients of $\Psi_{\Gamma}$ are all either 0 or +1 .

For the graph $\Gamma$, we build the table $\operatorname{Tab}(\Gamma)$ with $h(\Gamma)$ rows and $|E(\Gamma)|$ columns. Each row corresponds to a loop of $\Gamma$, and these loops form a basis of $H_{1}(\Gamma, \mathbb{Z})$. For each such loop we choose some direction of loop tracing. The entry $\operatorname{Tab}(\Gamma)_{i j}$ equals 1 if the edge $e_{j}$ in the $i$ 's loop is in the tracing direction of the loop and equals -1 if this edge is in the opposite direction; if the edge $e_{j}$ does not appear in the $i$ 's loop, then $\operatorname{Tab}(\Gamma)_{i j}=0$. We take 
$N=|E(\Gamma)|$ variables $T_{1}, \ldots, T_{N}$ and build a matrix

$$
\mathcal{M}_{\Gamma}(T)=\sum_{k=1}^{N} T_{k} \mathcal{M}^{k},
$$

where $\mathcal{M}^{k}$ is a $h_{1}(\Gamma) \times h_{1}(\Gamma)$ matrix with entries

$$
\mathcal{M}_{i j}^{d}=\operatorname{Tab}(\Gamma)_{i d} \cdot \operatorname{Tab}(\Gamma)_{j d}
$$

By definition, the graph polynomial for $\Gamma$ is

$$
\Psi_{\Gamma}(T)=\operatorname{det} \mathcal{M}_{\Gamma}(T)
$$

Consider the following example which will appear in Section 2.1.

Example 2.1. Let $\Gamma$ be the graph $\mathrm{ZZ}_{5}$ (see the drawing). This graph has 10 edges and the Betti number equals 5.
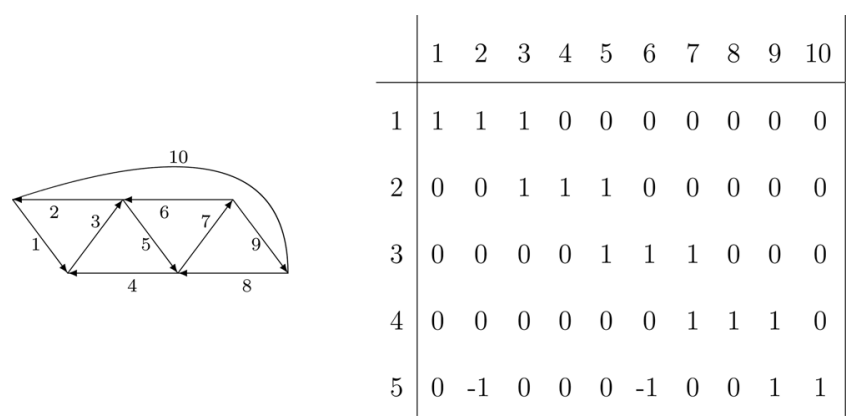

We choose the orientation and the numbering of edges as on the drawing to the left. Following the construction above, we build the table Tab $\left(Z_{5}\right)$ to the right and get the following matrix:

$$
\mathcal{M}_{\mathrm{ZZ}_{5}}(T)=\left(\begin{array}{ccccc}
T_{1}+T_{2}+T_{3} & T_{3} & 0 & 0 & -T_{2} \\
T_{3} & T_{3}+T_{4}+T_{5} & T_{5} & 0 & 0 \\
0 & T_{5} & T_{5}+T_{6}+T_{7} & T_{7} & -T_{6} \\
0 & 0 & T_{7} & T_{7}+T_{8}+T_{9} & T_{9} \\
-T_{2} & 0 & -T_{6} & T_{9} & T_{2}+T_{6}+T_{9}+T_{10}
\end{array}\right) .
$$

Definition 2.3. The graph hypersurface $X_{\Gamma} \subset \mathbb{P}^{N-1}$ is the hypersurface cut out by $\Psi_{\Gamma}=0$. 
Throughout the whole paper, we deal with such graph hypersurfaces. Sometimes it is convenient to make a linear change of coordinate in $\mathbb{P}^{N-1}$ to simplify the matrix. Clearly, this new matrix $\widetilde{\mathcal{M}}_{\Gamma}$ will define a hypersurface isomorphic to $X$, which we denote again by $X$. For the graph in Example 2.1 , we note that $T_{0}, T_{4}, T_{8}$ and $T_{10}$ appear only in the diagonal of $\mathcal{M}_{\mathrm{ZZ}_{5}}(T)$. Changing the coordinates and defining new variables, we get the matrix

$$
\mathcal{M}=\mathcal{M}_{Z Z_{5}}(A, B)=\left(\begin{array}{ccccc}
B_{0} & A_{0} & 0 & 0 & A_{5} \\
A_{0} & B_{1} & A_{1} & 0 & 0 \\
0 & A_{1} & C_{2} & A_{2} & A_{4} \\
0 & 0 & A_{2} & B_{3} & A_{3} \\
A_{5} & 0 & A_{4} & A_{3} & B_{4}
\end{array}\right)
$$

where $C_{2}=A_{1}+A_{2}-A_{4}$.

Definition 2.4. The graph $\Gamma$ is said to be convergent (resp. logarithmically divergent) if $N>2 h_{1}(\Gamma)$ (resp. $N=2 h_{1}(\Gamma)$ ). The logarithmically divergent graph $\Gamma$ is primitively log divergent if any connected proper subgraph $\Gamma^{\prime} \subset \Gamma$ is convergent.

For a primitively log divergent graph the quantity of interest is the period defined by

$$
P(\Gamma):=\int_{\sigma^{2 n-1}(\mathbb{R})} \eta_{\Gamma} \quad \text { with } \eta_{\Gamma}=: \frac{\Omega_{2 n-1}(A)}{\Psi_{\Gamma}^{2}}
$$

where $\sigma^{2 n-1}(\mathbb{R}) \subset \mathbb{P}^{2 n-1}(\mathbb{R})$ is a locus of points with non-negative coordinates and

$$
\Omega_{2 n-1}(A)=\sum_{i=1}^{2 n}(-1)^{i} A_{i} d A_{1} \wedge \ldots \widehat{d A_{i}} \cdots \wedge d A_{2 n}
$$

The integral converges (see [2, Proposition 5.2]).

In Section 7 of [2] a relative cohomology was constructed.

$$
H:=H^{2 n-1}(P \backslash Y, B \backslash B \cap Y),
$$

with period (i.e., the integration along a homology of $H$ with a de Rham cohomology of $H$ ) exactly (2.9). Here $P$ is some blowing up of $\mathbb{P}^{2 n-1}, Y$ is a the strict transform of $X_{\Gamma}$ and $B$ is the total transform of $A_{1} A_{2} \ldots A_{2 n}=0$. 
There is a hope (see $[2,7.25])$ that for all primitively log divergent graphs, or for an identifiable subset of them, the maximal weight piece of the Betti realization $H_{\mathrm{B}}$ is Tate:

$$
\operatorname{gr} \underset{\max }{W} H_{\mathrm{B}}=\mathbb{Q}(-p)^{\oplus r}
$$

One would like to find a rank 1 sub-Hodge structure $\iota: \mathbb{Q}(-p) \hookrightarrow \operatorname{gr} \max _{\mathrm{B}}^{W} H_{\mathrm{B}}$ such that the image of $\eta_{\Gamma}$ in $\operatorname{gr}_{\max }^{W} H_{\mathrm{DR}}$ spans $\iota(\mathbb{Q}(-p))_{\mathrm{DR}}$.

Unfortunately, we cannot compute this even in very simple cases, but something can be done here. Note that by the construction of the blowing up above, we have a natural inclusion $\mathbb{P}^{2 n-1} \backslash X \hookrightarrow P \backslash Y$. This implies a morphism

$$
H^{2 n-1}(P \backslash Y) \stackrel{j}{\rightarrow} H^{2 n-1}\left(\mathbb{P}^{2 n-1} \backslash X\right) .
$$

Furthermore, the relative cohomology in (2.11) fits into an exact sequence

$$
\longrightarrow H^{2 n-2}(B \backslash B \cap Y) \longrightarrow H \longrightarrow H^{2 n-1}(P \backslash Y) \longrightarrow \text {. }
$$

The idea (and the only thing we can do) is to compute $H^{2 n-1}\left(\mathbb{P}^{2 n-1} \backslash X\right)$. We hope that the map $j$ in (2.13) is nonzero, otherwise our computations give no information about $H$. In $\left[2\right.$, Section 11], $H^{2 n-1}\left(\mathbb{P}^{2 n-1} \backslash X_{n}\right) \cong \mathbb{Q}(-2 n+3)$ was computed for $X_{n}$, the graph hypersurface of $\mathrm{WS}_{n}, n \geq 3$ (for Betti or l-adic cohomology). Moreover, motivated by discussion above about the weights of realizations of $H$, for the de Rham cohomology it was proved (see Section 12) that the class of $\eta_{\Gamma}$ lies in the second level of the Hodge filtration (and generates the whole cohomology because $H_{\mathrm{DR}}^{2 n-1}\left(\mathbb{P}^{2 n-1} \backslash X_{n}\right)$ ) is one dimensional).

In the next sections we compute $H^{2 n-1}\left(\mathbb{P}^{2 n-1} \backslash X\right)$ (or the maximal graded piece of weight filtration) for new examples of primitively divergent graphs. For $\mathrm{ZZ}_{5}$, we also managed to do the computation for $H_{\mathrm{DR}}^{2 n-1}$ $\left(\mathbb{P}^{2 n-1} \backslash X\right)$.

\section{Cohomology}

In this section, we explain the cohomological tools we will use. By Corollary 2.1 , a graph hypersurface $X$ is always defined over $\mathbb{Z}$. We consider two types of cohomology theories: the $\ell$-adic cohomology $H^{i}\left(X \otimes_{\mathbb{Z}} \overline{\mathbb{Q}}, \mathbb{Q}_{\ell}\right)$ and the Betti cohomology $H^{i}\left(X \otimes_{\mathbb{Z}} \mathbb{C}, \mathbb{Q}\right)$. To unify the notation we simply write $H^{i}(X)$ and everything below works for both theories. 
Let $X$ be a proper scheme over some field $K$ of char. 0 and $Z \subset X$ a closed subscheme, one has the following exact localization sequence:

$$
\longrightarrow H_{\mathrm{c}}^{r}(X \backslash Z) \longrightarrow H^{r}(X) \longrightarrow H^{r}(Z) \longrightarrow,
$$

where $H_{\mathrm{c}}^{r}$ is the cohomology with compact support. We assume $K$ to be algebraically closed. We will use the following standart fact.

Theorem 3.1. Denote by $\operatorname{cd}(X)$ the cohomological dimension of a variety $X$. Then

- $\operatorname{cd}(X) \leq 2 \operatorname{dim}(X)$

- $\operatorname{cd}(X) \leq \operatorname{dim}(X)$ if $X$ is affine.

Applying the Poincaré duality in the case of a smooth affine $X$, we get dually $H_{\mathrm{c}}^{r}(X)=0$ for $r<\operatorname{dim}(X)$.

Let now $X \subset \mathbb{P}^{m}$ be defined by the vanishing of one homogeneous polynomial $f \in K\left[x_{0}, \ldots, x_{m}\right], m \geq 2$, we write $X=\mathcal{V}(f)$ in such situation. Applying (3.1) to the inclusion $X \hookrightarrow \mathbb{P}^{m}$, we get

$$
\longrightarrow H_{\mathrm{c}}^{r}\left(\mathbb{P}^{m} \backslash X\right) \longrightarrow H^{r}\left(\mathbb{P}^{m}\right) \longrightarrow H^{r}(X) \longrightarrow .
$$

But $\mathbb{P}^{m} \backslash X$ is affine (and smooth) of dimension $m$, thus by the Artin vanishing $H_{\mathrm{c}}^{r}\left(\mathbb{P}^{m} \backslash X\right)=0$ for $0 \leq r \leq m-1$. This implies $H^{r}(X) \cong H^{r}\left(\mathbb{P}^{m}\right)$ for $0 \leq r \leq m-2$ and $H^{m-1}\left(\mathbb{P}^{m}\right) \hookrightarrow H^{m-1}(X)$. So, the first interesting cohomology of a hypersurface in $\mathbb{P}^{m}$ is in degree $m-1$, we sometimes call it the cohomology of the middle degree $H^{\mathrm{mid}}(X)$.

Definition 3.1. Define $H_{\text {prim }}^{r}(X):=\operatorname{coker}\left(H^{r}\left(\mathbb{P}^{m}\right) \longrightarrow H^{r}(X)\right)$ for all $r$.

Theorem 3.2. Let $X \subset \mathbb{P}^{n}$ be a variety over algebraically closed field of characteristic 0 . Then the morphism

$$
\phi_{r}: H^{r}\left(\mathbb{P}^{n}\right) \longrightarrow H^{r}(X)
$$

is injective for $0 \leq r \leq 2 \operatorname{dim} X$.

Let $X$ be a proper scheme and $Y \subset X$ be a closed subscheme. By the theorem above, the localization sequence for $Y \subset X$ implies that the sequence

$$
\longrightarrow H_{\mathrm{c}}^{i}(X \backslash Y) \longrightarrow H_{\mathrm{prim}}^{i}(X) \longrightarrow H_{\mathrm{prim}}^{i}(Y) \longrightarrow
$$


is exact in all terms up to $H_{\text {prim }}^{i}(Y)$ for $i=2 \operatorname{dim} Y$. The Mayer-Vietoris sequence for the closed covering $X=X_{1} \cup X_{2}$ yields the sequence

$$
\longrightarrow H_{\text {prim }}^{i}(X) \longrightarrow H_{\text {prim }}^{i}\left(X_{1}\right) \oplus H_{\text {prim }}^{i}\left(X_{2}\right) \longrightarrow H_{\text {prim }}^{i}\left(X_{1} \cap X_{2}\right) \longrightarrow,
$$

which is exact in terms up to $H_{\text {prim }}^{i}\left(X_{1} \cap X_{2}\right)$ for $i=2 \operatorname{dim} X_{1} \cap X_{2}$.

The next two theorems are often referred to in the next sections.

Theorem 3.3 (Vanishing Theorem A). Let $Y$ be a variety $\mathcal{V}\left(f_{1}, f_{2}, \ldots\right.$, $\left.f_{k}\right) \subset \mathbb{P}^{N}\left(a_{0}: a_{1}: \ldots: a_{N}\right)$ for some homogeneous polynomials $f_{1}, \ldots, f_{k} \in$ $K\left[a_{0}, \ldots, a_{N}\right]$, and suppose that $f_{i}$ is independent of the first $t$ variables $a_{0}, \ldots, a_{t-1}$ for each $i, 1 \leq i \leq k$. Then

(1) $H_{\text {prim }}^{r}(Y)=0$ for $r<N-k+t$.

(2) $H^{r}(Y) \cong H^{r-2 t}\left(Y^{\prime}\right)(-t)$ for $r \geq 2 t$, where $Y^{\prime} \subset \mathbb{P}^{N-t}$ is defined by the same polynomials.

Theorem 3.4 (Vanishing Theorem B). For homogeneous polynomials $f_{1}, \ldots, f_{k}, h \in K\left[a_{0}, \ldots, a_{N}\right], k \geq 0$, define a subscheme $U \subset \mathbb{P}^{N}$ by equations $f_{1}=\ldots=f_{k}=0$ and inequality $h \neq 0$, i.e.,

$$
U:=\mathcal{V}\left(f_{1}, \ldots, f_{k}\right) \backslash \mathcal{V}\left(f_{1}, \ldots, f_{k}, h\right) .
$$

Suppose that all the polynomials are independent of the first $t$ variables $a_{0}, \ldots, a_{t-1}$, and let $U^{\prime} \subset \mathbb{P}^{N-t}$ be defined by the same polynomials but in $\mathbb{P}^{N-t}\left(a_{t}: \ldots: a_{N}\right)$. Then

(1) $H_{\mathrm{c}}^{i}(U)=0$ for $i<N-k+t$.

(2) $H_{\mathrm{c}}^{i}(U) \cong H_{\mathrm{c}}^{i-2 t}\left(U^{\prime}\right)(-t)$.

Remark 3.1. In the computation for GZZ (Theorem 4.2) we do not want to apply the Artin vanishing. So we use only the homotopy invariance (from now h.i. everywhere), this means, we use only the second parts of Theorem A and Theorem B. This makes our proof more motivic, since one does not have the Artin vanishing for motivic cohomology.

\section{Generalized zigzag graphs}

Definition 4.1. Fix some $t \geq 1$ and consider a set $V(\Gamma)$ of $t+2$ vertexes $u_{i}, 1 \leq i \leq t+2$. Define $p\left(u_{1}, \ldots, u_{t+2}\right)$ to be the set of $t+1$ edges $\left(u_{i}, u_{i+1}\right)$, 
$1 \leq i \leq t+1$. Let $E(\Gamma):=p\left(u_{1}, \ldots, u_{t+2}\right)$. Now choose some positive integers $l_{i}$ for $1 \leq i \leq t$ with $l_{1} \geq 2$ and $l_{t} \geq 2$. For each $i, 1 \leq i \leq t$, we add $l_{i}-1$ new vertexes $v_{i j}, 1 \leq j \leq l_{i}-1$, and $l_{i}$ new edges $p\left(u_{i}, v_{i 1}, \ldots, v_{i l_{i}-1}, u_{i+2}\right)$, and $l_{i}-1$ edges $\left(v_{i j}, u_{i+1}\right), 1 \leq j \leq l_{i}-1$. Finally, we add an edge $\left(u_{1}, u_{t+2}\right)$. We call the constructed graph $\Gamma=(V(\Gamma), E(\Gamma))$ the generalized zigzag graph $\operatorname{GZZ}\left(l_{1}, \ldots, l_{t}\right)$.

For $\operatorname{GZZ}\left(l_{1}, \ldots, l_{t}\right)$ we define $n=1+\sum_{i=1}^{t} l_{i}$. The graph $\operatorname{GZZ}\left(l_{1}, \ldots, l_{t}\right)$ has $n+1$ vertexes, $2 n$ edges and the Betti number equals $n$. Thus, $\operatorname{GZZ}\left(l_{1}, \ldots, l_{t}\right)$ is a logarithmically divergent graph.

Example 4.1. The graph $\operatorname{GZZ}(3,2,3,4)$ looks like

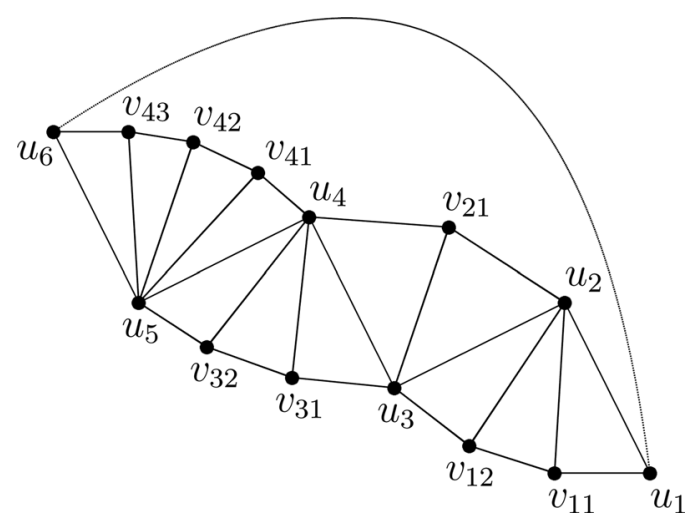

Example 4.2. The wheel with spokes graph $\mathrm{WS}_{n}$ is isomorphic to the generalized zigzag graph $\operatorname{GZZ}(n-1), n \geq 3$.

Example 4.3. The zigzag graph $\mathrm{ZZ}_{n}$ is isomorphic to the $\mathrm{GZZ}(2,1, \ldots$, 1,2 ) (with $n-51$ 's in the middle) for $n \geq 5$.

Theorem 4.1. A generalized zigzag graph $\Gamma=\operatorname{GZZ}\left(l_{1}, \ldots, l_{t}\right)$ is primitively log divergent.

Proof. We need to prove that for any proper subgraph $\Gamma^{\prime} \subset \Gamma$ the inequality $\left|E\left(\Gamma^{\prime}\right)\right|>2 h_{1}\left(\Gamma^{\prime}\right)$ holds, which means that $\Gamma^{\prime}$ is convergent. We do not distinguish between a graph and its set of edges. Because our graph $\Gamma$ is planar, it partitions the plain into exactly $h_{1}+1$ pieces. This is a good way to compute $h_{1}$. We will call the loops of length 3 simple loops. We can order the simple loops from the right bottom corner to the left top keeping in mind 
the drawing like in Example 4.1. Formally, let $\Delta_{1}=p\left(u_{1}, u_{2}, v_{11}, u_{1}\right)$ and for each $i$ we define the next simple loop $\Delta_{i+1}$ to be a simple loop, which has a common edge with $\Delta_{i}$ but not already labeled. Define $\Gamma_{0}:=\Gamma \backslash\left(u_{1}, u_{t+2}\right)$. The main point of the proof is the following. The graph $\Gamma_{0}$ is a strip of $\Delta$ 's, for each i, $1 \leq i \leq k$, we can cut this strip along $\left(u_{i}, u_{i+1}\right)$, turn over one piece and glue again along the same edges. Denote this operation by $\phi_{i}$. This gives a map

$$
\phi:=\phi_{t} \circ \ldots \circ \phi_{2}: \Gamma_{0} \longrightarrow \hat{\Gamma}_{0}
$$

where $\hat{\Gamma}_{0}$ is isomorphic to $\mathrm{WS}_{n}$ without one boundary edge; this graph is topologically the same as a half of $\mathrm{WS}_{n}$, we denote it by $\mathrm{hWS}_{n}$. Note that the maps $\phi_{i}$ and $\phi$ are the isomorphisms between sets of edges of the graphs in the described way. On some vertexes this map is not single-valued. For the graph $\Gamma$ in Example 4.1, we have the following $\hat{\Gamma}_{0}=\mathrm{hWS}_{13}$ :

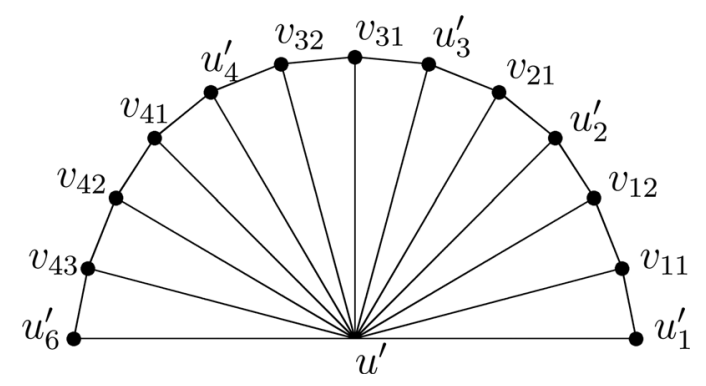

The vertex $u_{i}$ under described operations goes to $u_{i-1}^{\prime}$, or $u_{i}^{\prime}$, or $u^{\prime}$ depending on the edge that we take. We can label the simple loops of $\mathrm{hWS}_{n}$ from the right to the left by $\hat{\Delta}_{1}, \ldots, \hat{\Delta}_{n-1}$, these are the images of $\Delta$ 's

$$
\phi\left(\Delta_{i}\right)=\hat{\Delta}_{i}
$$

Each $\phi_{i}$ preserves loops; this means that a subgraph $\gamma \subset \phi_{i-1} \ldots \phi_{2}(\Gamma)$ is a loop if and only if $\phi_{i}(\gamma)$ is a loop of the same length. Thus, this condition holds for $\phi$. It follows that $\Gamma_{0}$ and $\mathrm{hWS}_{n}$ have the same Betti numbers. Moreover, for each subgraph $\Gamma_{0}^{\prime \prime} \subset \Gamma_{0}$ we have

$$
h_{1}\left(\Gamma_{0}^{\prime \prime}\right)=h_{1}\left(\phi\left(\Gamma_{0}^{\prime \prime}\right)\right)
$$


To involve the "special" edge $\left(u_{1}, u_{t+2}\right)$ into consideration, note that if the graph $\Gamma_{0}^{\prime \prime}$ is disconnected and we have no path $p^{\prime}\left(u_{1}, \ldots, u_{t+1}\right)$ with endpoints $u_{1}$ and $u_{t+1}$, then the adding of $\left(u_{1}, u_{t+2}\right)$ does not change the Betti number; otherwise this increases the number by one.

$$
h\left(\Gamma_{0}^{\prime \prime} \cup\left(u_{1}, u_{t+1}\right)\right)= \begin{cases}h\left(\Gamma_{0}^{\prime \prime}\right), & p^{\prime}\left(u_{1}, \ldots, u_{t+2}\right) \not \subset \Gamma_{0}^{\prime \prime}, \\ h\left(\Gamma_{0}^{\prime \prime}\right)+1, & \text { otherwise }\end{cases}
$$

This proves that we can extend the map $\phi$ to

$$
\bar{\phi}: \Gamma \longrightarrow \hat{\Gamma}
$$

which maps our graph to $\hat{\Gamma}$, that is nothing but $\hat{\Gamma}_{0} \cong \mathrm{hWS}_{n}$ compactified by adding the missing boundary edge and is isomorphic to $\mathrm{WS}_{n}$. The map $\bar{\phi}$ satisfies the same condition as $\phi$ in (4.3). For the example of $\mathrm{hWS}_{13}$ above, we add the edge $\left(u_{1}, u_{6}\right)$ on the drawing and get $\mathrm{WS}_{13}$.

So, we reduced the statement to the case $\mathrm{WS}_{n}$. It is known that these graphs are primitively log divergent. This concludes the proof.

Let $X \subset \mathbb{P}^{2 n-1}$ be a graph hypersurface. We consider the Betti cohomology of the middle degree $H^{\text {mid }}(X)=H^{2 n-2}(X)$. By Deligne's theory of MHS $[5,6]$, there is a $\mathbb{Q}$-mixed Hodge structure associated to $H^{\operatorname{mid}}(X)$. We can try to study the graded pieces of weight filtration $W: \operatorname{gr}_{i}^{W}\left(H^{r}(X)\right), 0 \leq i \leq$ $2 n-2, r \geq$ mid.

Theorem 4.2. For the hypersurface $X$ associated to a generalized zigzag graph GZZ, one has the isomorphisms

$$
\begin{array}{r}
\operatorname{gr}_{4}^{W}\left(H_{\text {prim }}^{\operatorname{mid}}(X)\right) \cong W_{4}\left(H_{\text {prim }}^{\operatorname{mid}}(X)\right) \cong W_{5}\left(H_{\text {prim }}^{\operatorname{mid}}(X)\right) \cong \mathbb{Q}(-2), \\
W_{5}\left(H_{\text {prim }}^{r}(X)\right)=0, \quad r>\text { mid }
\end{array}
$$

Proof. We consider the case when $t$ is even and start with labeling of edges and choosing orientations. For simplicity, let $n_{0}:=0$ and

$$
n_{i}:=\sum_{j=1}^{i} l_{j} \text { for } 1 \leq i \leq t
$$


For each $i, 1 \leq i \leq t$, define $e_{n_{i-1}+1}:=\left(u_{i+1}, u_{i}\right)$ for odd $i$ and $e_{n_{i-1}+1}:=$ $\left(u_{i}, u_{i+1}\right)$ for even $i$,

$$
e_{n_{i-1}+j}:= \begin{cases}\left(u_{i+1}, v_{i j-1}\right) & \text { for } 2 \leq j \leq l_{i}, \quad i \text { odd } \\ \left(v_{i j-1}, u_{i+1}\right) & \text { for } 2 \leq j \leq l_{i}, \quad i \text { even }\end{cases}
$$

Together with $e_{n_{t}+1}:=\left(u_{t+2}, u_{t+1}\right)$ for even $t$ and $e_{n_{t}+1}:=\left(u_{t+1}, u_{t+2}\right)$ for odd $t$, these are the first $n_{t}+1=: n$ edges. Now, for each $i, 1 \leq i \leq t$, define $e_{n+n_{i-1}+1}:=\left(v_{i 1}, u_{i}\right)$,

$$
e_{n+n_{i-1}+j}:=\left(v_{i j}, v_{i j-1}\right) \text { for } 2 \leq j \leq l_{i}-1
$$

and $e_{n+n_{i-1}+l_{i}}:=\left(u_{i+2}, v_{i l_{i}-1}\right)$. Roughly speaking, all edges are oriented from the left top corner to the right bottom and from the right top corner to the left bottom corner. Define $e_{2 n}:=\left(u_{1}, u_{t+2}\right)$.

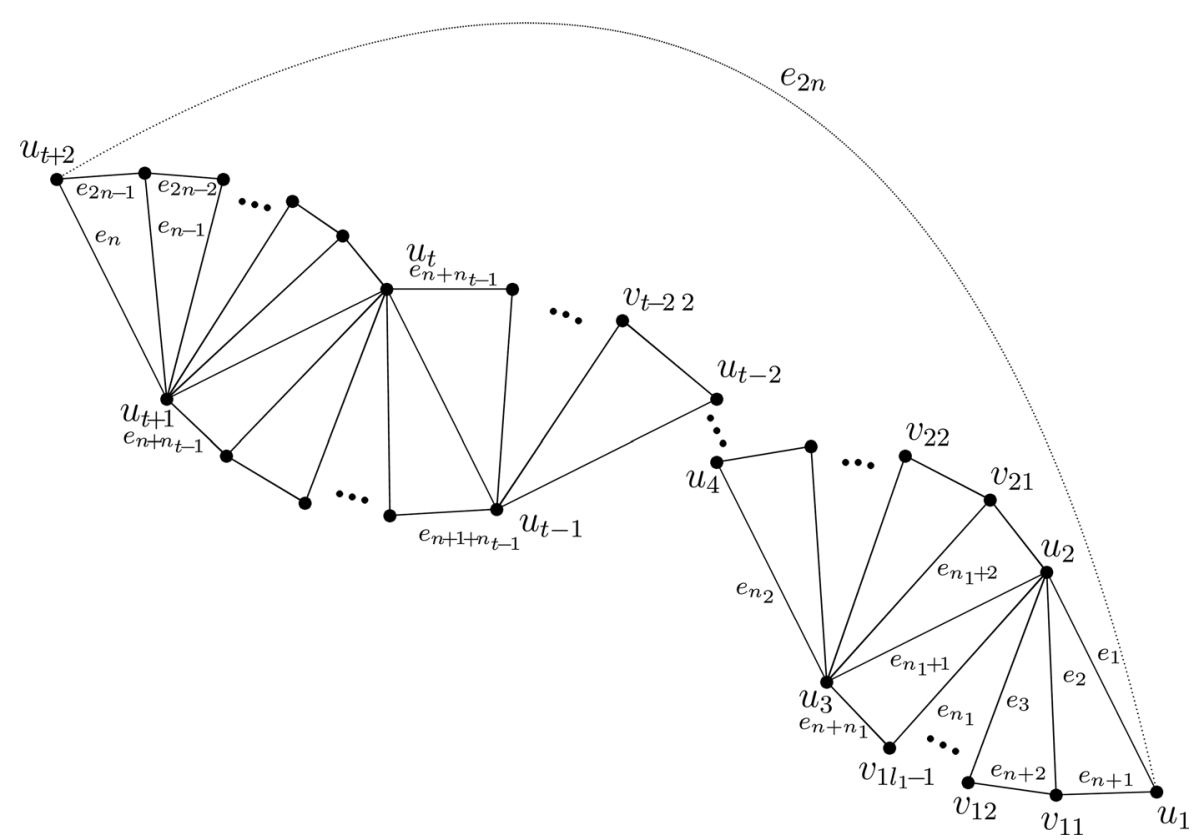




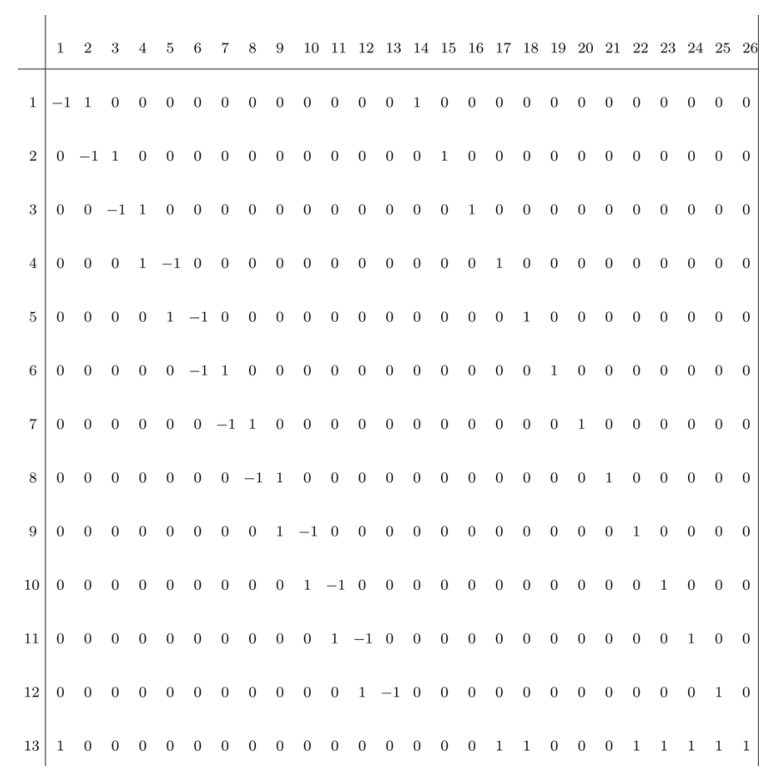

For building the table, we take the small loops from the right bottom corner of the drawing to the left top corner, and the last loop to be chosen is the loop with the edge $\left(u_{1}, u_{t+2}\right)$. Because of lack of space, we draw the table for the graph in Example 4.1.

Now we take $2 n$ variables $T_{1}, \ldots, T_{2 n}$ and build a matrix $\mathcal{M}(T)$ as the sum of elementary matrices. After a change of the variables, we get the matrix

$$
\mathcal{M}_{\mathrm{GZZ}}=\left(\begin{array}{ccccccccccccc}
B_{0} & A_{0} & 0 & 0 & 0 & 0 & 0 & 0 & 0 & 0 & 0 & 0 & A_{17} \\
A_{0} & B_{1} & A_{1} & 0 & 0 & 0 & 0 & 0 & 0 & 0 & 0 & 0 & 0 \\
0 & A_{1} & B_{2} & A_{2} & 0 & 0 & 0 & 0 & 0 & 0 & 0 & 0 & 0 \\
0 & 0 & A_{2} & C_{3} & A_{3} & 0 & 0 & 0 & 0 & 0 & 0 & 0 & A_{16} \\
0 & 0 & 0 & A_{3} & C_{4} & A_{4} & 0 & 0 & 0 & 0 & 0 & 0 & A_{15} \\
0 & 0 & 0 & 0 & A_{4} & B_{5} & A_{5} & 0 & 0 & 0 & 0 & 0 & 0 \\
0 & 0 & 0 & 0 & 0 & A_{5} & B_{6} & A_{6} & 0 & 0 & 0 & 0 & 0 \\
0 & 0 & 0 & 0 & 0 & 0 & A_{6} & B_{7} & A_{7} & 0 & 0 & 0 & 0 \\
0 & 0 & 0 & 0 & 0 & 0 & 0 & A_{7} & C_{8} & A_{8} & 0 & 0 & A_{14} \\
0 & 0 & 0 & 0 & 0 & 0 & 0 & 0 & A_{8} & C_{9} & A_{9} & 0 & A_{13} \\
0 & 0 & 0 & 0 & 0 & 0 & 0 & 0 & 0 & A_{9} & C_{10} & A_{10} & A_{12} \\
0 & 0 & 0 & 0 & 0 & 0 & 0 & 0 & 0 & 0 & A_{10} & B_{11} & A_{11} \\
A_{17} & 0 & 0 & A_{16} & A_{15} & 0 & 0 & 0 & A_{14} & A_{13} & A_{12} & A_{11} & B_{12}
\end{array}\right) .
$$


In the last row the $A$ 's appear in the zero column and in the columns $n_{i}+$ $j-1$ for all $i \not \equiv t \bmod 2,1 \leq i \leq t$, and all $1 \leq j \leq l_{i}$. In the same columns (but 0 and $n-2$ ) we have $C$ 's in the main diagonal. These $C$ 's are defined by

$$
C_{k}:= \begin{cases}A_{v}+A_{k-1}-A_{k}, \quad k=n_{i}, l_{i+1}>1, \quad i \neq 0 \\ A_{v}-A_{k-1}-A_{k}, \quad k=n_{i}+j, \quad 1 \leq j \leq l_{i+1}-2, \\ A_{v}-A_{k-1}+A_{k}, \quad k=n_{i+1}-1, \quad l_{i+1}>1, i \neq t-1, \\ A_{v}+A_{k-1}+A_{k}, \quad k=n_{i}, \quad l_{i+1}=1,\end{cases}
$$

where $i \not \equiv t \bmod 2$, and $A_{v}$ is always in the last row in the same column as $C_{k}$. Formally, if $k=n_{i}+j-1$, then

$$
v=v(k)=n-2+\sum_{\substack{r=i+2 \\ r \neq t \bmod 2}}^{t-1} l_{r}+l_{i+1}-j .
$$

In the computations we think of $C_{k}$ as just linear polynomials of $A$ 's rather than entries of the matrix. Sometimes we denote by $A_{m}$ the entry in the left bottom corner of $\mathcal{M}_{\mathrm{GZZ}}$.

For the case of odd $t$ we can derive the tables and the matrices from the even case. Indeed, consider some $\Gamma^{\prime}=\operatorname{GZZ}\left(l_{1}, \ldots, l_{t}\right)$ with even $t$ and let $\Gamma$ be the graph which we get from $\Gamma^{\prime}$ after forgetting edges of simple loops $\Delta_{1}, \ldots, \Delta_{l_{1}}$ (see Theorem 4.1 for definition), we assume that $\left(u_{2}, u_{3}\right)$ remains, and we take $\left(u_{t+2}, u_{2}\right)$ instead of $\left(u_{t+2}, u_{1}\right)$. So, $\Gamma=\operatorname{GZZ}\left(l_{2}, \ldots, l_{t}\right)$. Constructing everything similar, the table for $\Gamma$ is that for $\Gamma^{\prime}$ without first $l_{1}$ rows. The matrix of $\Gamma$ looks similar to that of $\Gamma^{\prime}$ with the same assumptions on $A$ 's in the last row and on $C$ 's.

Consider the projective space $\mathbb{P}^{2 n-1}$ with coordinates all the $A_{i}$ 's and $B_{j}$ 's appearing in the matrix and define $X:=\mathcal{V}\left(\operatorname{det}\left(\mathcal{M}_{\mathrm{GZZ}}\right)\right)=\mathcal{V}\left(I_{n}\right) \subset$ $\mathbb{P}^{2 n-1}$, where

$$
\mathcal{M}_{\mathrm{GZZ}}=\left(\begin{array}{cccc}
\ddots & \vdots & \vdots & \vdots \\
\cdots & C_{n-3} & A_{n-3} & A_{n-1} \\
\cdots & A_{n-3} & B_{n-2} & A_{n-2} \\
\cdots & A_{n-1} & A_{n-2} & B_{n-1}
\end{array}\right)
$$

Since $l_{t}>1$, the entry $a_{n-3 n-3}$ is really not independent, and thus $C_{n-3}$. 
Step 1. Fix some $r, 2 n-2 \leq r \leq 4 n-4$. We are going to compute $H^{r}(X)$. For the closed subscheme $\mathcal{V}\left(I_{n}, I_{n-1}\right) \subset X$, we have the localization sequence

$$
\begin{aligned}
\rightarrow H_{c}^{r}\left(X \backslash \mathcal{V}\left(I_{n}, I_{n-1}\right)\right) \rightarrow H^{r}(X) \rightarrow \\
\quad H^{r}\left(\mathcal{V}\left(I_{n}, I_{n-1}\right)\right) \rightarrow H_{\mathrm{c}}^{r+1}\left(X \backslash \mathcal{V}\left(I_{n}, I_{n-1}\right)\right) \rightarrow .
\end{aligned}
$$

We can write

$$
I_{n}=B_{n-1} I_{n-1}-G_{n-1},
$$

where $G_{n-1}$ is independent of $B_{n-1}$. Projecting from the point where all the variables but $B_{n-1}$ are zero, we get

$$
X \backslash \mathcal{V}\left(I_{n}, I_{n-1}\right) \cong \mathbb{P}^{2 n-2} \backslash \mathcal{V}\left(I_{n-1}\right)
$$

Since $I_{n-1}$ is independent of $A_{n-2}$ and $A_{m}$, the h.i. for scheme on the righthand side of (4.16) implies

$$
H_{\mathrm{c}}^{q}\left(X \backslash \mathcal{V}\left(I_{n}, I_{n-1}\right)\right) \cong H_{\mathrm{c}}^{q-4}\left(\mathbb{P}^{2 n-4} \backslash \mathcal{V}\left(I_{n-1}\right)\right)(-2)
$$

for $q=r$ and $r+1$. Since $I_{n-1}$ is the determinant of the matrix without the last row and the last column, we see that the variables of the form $A_{v}$ appear in $I_{n-1}$ only inside the linear polynomial $C_{k}$ (see definition (4.11)). Taking a suitable change of variables (like $A_{v} \mapsto A_{v}-A_{k-1}+A_{k}$ in the first case of (4.11)), we get that the polynomial $C_{k}$ as an independent variable instead of $A_{v}$. We write usually $C_{k}:=A_{v}$ in such situation. So, we define by $I_{n-1}^{\prime}$ the image of $I_{n-1}$ under the change of the coordinates of $\mathbb{P}^{2 n-4}$ (no $\left.B_{n-1}, A_{n-2}, A_{m}\right) C_{i}:=A_{v(i)}$ for all $C_{i}$. Using the localization sequence for the closed embedding $I_{n-1}^{\prime} \subset \mathbb{P}^{2 n-4}$, we get

$$
H_{\mathrm{c}}^{q-4}\left(\mathbb{P}^{2 n-4} \backslash \mathcal{V}\left(I_{n-1}^{\prime}\right)\right) \cong H_{\text {prim }}^{q-5}\left(\mathcal{V}\left(I_{n-1}^{\prime}\right)\right) .
$$

Consider $T:=\mathcal{V}\left(I_{n-1}^{\prime}, I_{n-2}^{\prime}\right) \subset \mathcal{V}\left(I_{n-1}^{\prime}\right)$. One has the localization sequence

$$
\rightarrow H_{\mathrm{c}}^{q-5}\left(\mathcal{V}\left(I_{n-1}^{\prime}\right) \backslash T\right) \rightarrow H_{\text {prim }}^{q-5}\left(\mathcal{V}\left(I_{n-1}^{\prime}\right)\right) \rightarrow H_{\text {prim }}^{q-5}(T) \rightarrow .
$$

We can rewrite $T=\mathcal{V}\left(I_{n-2}^{\prime}, A_{n-3} I_{n-3}^{\prime}\right)$. So, the defining polynomials of $T$ do not depend on $B_{n-2}$. Now, on $\mathcal{V}\left(I_{n-1}^{\prime}\right) \backslash T$ we can express $B_{n-2}$ from the equation and get $\mathcal{V}\left(I_{n-1}^{\prime}\right) \backslash T \cong \mathbb{P}^{2 n-5} \backslash \mathcal{V}\left(I_{n-2}^{\prime}\right)$. The polynomial $I_{n-2}^{\prime}$ is independent of $A_{n-3}$. Thus, we can apply h.i. to $T$ and $\mathcal{V}\left(I_{n-1}^{\prime}\right) \backslash T$, and then 
applying gr $_{i}^{W}$ to (4.19), we obtain

$$
\operatorname{gr}_{i}^{W} H_{\mathrm{prim}}^{q-5}\left(\mathcal{V}\left(I_{n-1}^{\prime}\right)\right)=0, \quad i=0,1, \quad \text { any } q .
$$

By (4.17), (4.18) and (4.20), the sequence (4.14) yields

$$
\operatorname{gr}_{i}^{W} H^{r}(X) \cong \operatorname{gr}_{i}^{W} H^{r}\left(\mathcal{V}\left(I_{n}, I_{n-1}\right)\right), \quad i=0, \ldots, 5 .
$$

By (4.15), one has $\mathcal{V}\left(I_{n}, I_{n-1}\right) \cong \mathcal{V}\left(I_{n-1}, G_{n-1}\right)^{(2 n-1)}$. Both polynomials to the right are independent of $B_{n-1}$. H.i. for $\mathcal{V}\left(I_{n-1}, G_{n-1}\right)^{(2 n-1)}$ and (4.21) imply

$$
\operatorname{gr}_{i}^{W} H^{r}(X) \cong \operatorname{gr}_{i-2}^{W} H^{r-2}\left(\mathcal{V}\left(I_{n-1}, G_{n-1}\right)\right), \quad i=0, \ldots, 5
$$

The variety to the right lives in $\mathbb{P}^{2 n-2}$ (no $B_{n-1}$ ). Define the closed subscheme $\hat{V} \subset \mathcal{V}\left(I_{n-1}, G_{n-1}\right)$ by $\hat{V}:=\mathcal{V}\left(I_{n-1}, I_{n-2}, G_{n-1}\right) \subset \mathbb{P}^{2 n-2}\left(\right.$ no $\left.B_{n-1}\right)$. One has an exact sequence

$$
\begin{aligned}
\rightarrow & H_{\mathrm{c}}^{r-2}\left(\mathcal{V}\left(I_{n-1}, G_{n-1}\right) \backslash \hat{V}\right) \rightarrow H^{r-2}\left(\mathcal{V}\left(I_{n-1}, G_{n-1}\right)\right) \rightarrow \\
& H^{r-2}(\hat{V}) \rightarrow H_{\mathrm{c}}^{r-1}\left(\mathcal{V}\left(I_{n-1}, G_{n-1}\right) \backslash \hat{V}\right) \rightarrow .
\end{aligned}
$$

The polynomial $I_{n-1}$ is independent of $A_{n-2}$ and the coefficient of $A_{n-2}^{2}$ in $G_{n-1}$ is $I_{n-2}$. By Corollory 1.3 , we have $I_{n-2} G_{n-1}=\left(L_{n-1}\right)^{2}$ on $\mathcal{V}\left(I_{n-1}\right)$, so

$$
\left\{\begin{array} { r } 
{ I _ { n - 1 } = G _ { n - 1 } = 0 , } \\
{ I _ { n - 2 } \neq 0 , }
\end{array} \Leftrightarrow \left\{\begin{array}{r}
I_{n-1}=L_{n-1}=0, \\
I_{n-2} \neq 0 .
\end{array}\right.\right.
$$

Now,

$$
L_{n-1}=A_{n-2} I_{n-2}+\sum_{s}(-1)^{s+n-2} A_{v}(s) I_{n-1}(s ; n-2),
$$

where the sum goes over all $s$ such that $a_{s s}=C_{s}$, and also $s=0$ (assuming $v(0)=m)$, see $(4.12),(1.11)$. Solving on $A_{n-2}$ and projecting from the point where all the coordinates but $A_{n-2}$ are zero, we get

$$
\mathcal{V}\left(I_{n-1}, G_{n-1}\right) \backslash \hat{V} \cong \mathcal{V}\left(I_{n-1}\right) \backslash \mathcal{V}\left(I_{n-1}, I_{n-2}\right) .
$$

Expressing $B_{n-2}$ from $I_{n-1}=0$ and projecting further, we obtain an isomorphism $\mathcal{V}\left(I_{n-1}\right) \backslash \mathcal{V}\left(I_{n-1}, I_{n-2}\right) \cong \mathbb{P}^{2 n-4} \backslash \mathcal{V}\left(I_{n-2}\right)$. The polynomial $I_{n-2}$ is independent of $A_{m}$ and $A_{n-1}$ (after $C_{n-3}:=A_{n-3}$ ). Applying $h$.i., one gets 
$\operatorname{gr}_{i}^{W} H_{\mathrm{c}}^{q}\left(\mathcal{V}\left(I_{n-1}, G_{n-1}\right) \backslash \hat{V}\right) \cong \operatorname{gr}_{i-4}^{W} H_{\mathrm{c}}^{q-4}\left(\mathbb{P}^{2 n-6} \backslash \mathcal{V}\left(I_{n-2}\right)\right)=0$ for $i=0, \ldots$, 3 and any $q$. The sequence (4.23) yields

$$
\operatorname{gr}_{i}^{W} H^{r-2}\left(\mathcal{V}\left(I_{n-1}, G_{n-1}\right)\right) \cong \operatorname{gr}_{i}^{W} H^{r-2}(\hat{V}), \quad i=0, \ldots, 3 .
$$

By Theorem 1.3, the polynomial $G_{n-1}$ is independent of $A_{n-2}$ on $\hat{V}$. Thus, $\hat{V}$ is defined by the vanishing of three polynomials which are independent of $A_{n-2}$. Applying h.i., one gets

$$
H^{r-2}(\hat{V}) \cong H^{r-4}(V)(-1),
$$

where $V:=\mathcal{V}\left(I_{n-1}, I_{n-2}, G_{n-1}^{\prime}\right) \subset \mathbb{P}^{2 n-3}$ (no $\left.B_{n-1}, A_{n-1}\right)$ and

$$
G_{n-1}^{\prime}:=\left.G_{n-1}\right|_{A_{n-2}=0} .
$$

Combining (4.22), (4.27) and (4.28), we get

$$
\operatorname{gr}_{i}^{W} H^{r}(X) \cong \operatorname{gr}_{i-4}^{W} H^{r-4}(V), \quad i=0, \ldots, 5 .
$$

Step 2. Now we get rid of $B_{n-2}$. We can write

$$
G_{n-1}^{\prime}=B_{n-2} G_{n-2}-A_{n-3}^{2} G_{n-3},
$$

where $G_{n-2}$ and $G_{n-3}$ are considered to be polynomials of variables $A_{n-1}, \ldots, A_{m}$ and $A_{n}, \ldots, A_{m}$ with "coefficients" from the Matrices of $I_{n-2}$ and of $I_{n-3}$, respectively. The decomposition follows from the fact that each coefficient of $G_{n-1}^{\prime}$ is a factor of some $I_{n-j-1}^{j}$ for $0 \leq j \leq n-2$, and the 3-diagonal matrix of $I_{n-j-1}^{j}$ has the right bottom entry $B_{n-2}$. Define the variety $\left.\hat{T}_{n-2}:=V \cap \mathcal{V}\left(G_{n-2}\right)=\mathcal{V}\left(A_{n-3} I_{n-3}, I_{n-2}, G_{n-2}, A_{n-3} G_{n-3}\right)\right) \subset$ $V$. One has an exact sequence

$$
H_{\text {prim }}^{r-5}(\hat{T}) \rightarrow H_{c}^{r-4}(V \backslash \hat{T}) \rightarrow H_{\text {prim }}^{r-4}(V) \rightarrow H_{\text {prim }}^{r-4}(\hat{T}) \rightarrow .
$$

Since the defining polynomials of $\hat{T}$ are independent of $B_{n-2}$, h.i. for $\hat{T}$ implies

$$
\rightarrow H_{\mathrm{prim}}^{r-7}(T)(-1) \rightarrow H_{\mathrm{c}}^{r-4}(V \backslash \hat{T}) \rightarrow H_{\mathrm{prim}}^{r-4}(V) \rightarrow H_{\mathrm{prim}}^{r-6}(T)(-1) \rightarrow
$$

for $T \subset \mathbb{P}^{2 n-4}$ (no $B_{n-1}, A_{n-2}, B_{n-2}$ ) defined by the same equations as $\hat{T}$. Applying the exact functor $\mathrm{gr}_{*}^{W}$ to the sequence above, we obtain

$$
\operatorname{gr}_{i}^{W} H_{\operatorname{prim}}^{r-4}(V) \cong \operatorname{gr}_{i}^{W} H_{\mathrm{c}}^{r-4}(V \backslash \hat{T}), \quad i=0,1 .
$$


The subscheme $V \backslash \hat{T} \subset V$ is defined by the system

$$
\left\{\begin{array}{r}
I_{n-2}=A_{n-3} I_{n-3}=0 \\
B_{n-2} G_{n-2}-A_{n-3}^{2} G_{n-3}=0 \\
G_{n-2} \neq 0 .
\end{array}\right.
$$

Projecting from the point where all the variables but $B_{n-2}$ are zero and solving the middle equation on $B_{n-2}$, we get an isomorphism

$$
\begin{aligned}
& V \backslash \hat{T} \cong \mathcal{V}\left(I_{n-2}, A_{n-3} I_{n-3}\right) \backslash \mathcal{V}\left(I_{n-2}, A_{n-3} I_{n-3}, G_{n-2}\right) \\
& \quad=: U_{1} \subset \mathbb{P}^{2 n-4}\left(\text { no } B_{n-1}, A_{n-2}, B_{n-2}\right) .
\end{aligned}
$$

One has an exact sequence

$$
\begin{aligned}
& H_{\mathrm{prim}}^{r-5}\left(\mathcal{V}\left(I_{n-2}, A_{n-3} I_{n-3}\right)\right) \rightarrow H_{\mathrm{prim}}^{r-5}\left(\mathcal{V}\left(I_{n-2}, A_{n-3} I_{n-3}, G_{n-2}\right)\right) \rightarrow \\
& \quad H_{\mathrm{c}}^{r-4}\left(U_{1}\right) \rightarrow H_{\mathrm{prim}}^{r-4}\left(\mathcal{V}\left(I_{n-2}, A_{n-3} I_{n-3}\right)\right) \rightarrow .
\end{aligned}
$$

The variety $\mathcal{V}\left(I_{n-2}, A_{n-3} I_{n-3}\right) \subset \mathbb{P}^{2 n-4}$ is defined by the polynomials independent of $A_{m}$. After applying of $h . i$. and then $\mathrm{gr}_{*}^{W}$, the sequence yields

$$
\operatorname{gr}_{i}^{W} H_{\mathrm{c}}^{r-4}\left(U_{1}\right) \cong \operatorname{gr}_{i}^{W} H_{\mathrm{prim}}^{r-5}\left(\mathcal{V}\left(I_{n-2}, A_{n-3} I_{n-3}, G_{n-2}\right)\right), \quad i=0,1
$$

Define $\hat{S}:=\mathcal{V}\left(I_{n-2}, I_{n-3}, G_{n-2}\right)$ and $U_{2}:=\mathcal{V}\left(I_{n-2}, A_{n-3} I_{n-3}, G_{n-2}\right) \backslash \hat{S}$ in $\mathbb{P}^{2 n-4}$. One has an exact sequence

$$
\begin{aligned}
\longrightarrow & H_{\mathrm{prim}}^{r-6}(\hat{S}) \longrightarrow H_{c}^{r-5}\left(U_{2}\right) \longrightarrow \\
& H_{\mathrm{prim}}^{r-5}\left(\mathcal{V}\left(I_{n-2}, A_{n-3} I_{n-3}, G_{n-2}\right)\right) \longrightarrow H_{\mathrm{prim}}^{r-5}(\hat{S}) \longrightarrow
\end{aligned}
$$

The only appearance of $A_{n-3}$ in the defining polynomials of $S$ is in $G_{n-2}$, namely in $C_{n-3}$. After a linear change of the variables we may assume that $C_{n-3}:=A_{n-3}$ is independent. Furthermore, the same argument as for $\hat{V}$ at Step 1 (see (4.28)) gives us $H^{r-5}(\hat{S}) \cong H^{r-7}(S)(-1)$ with $S:=$ $\mathcal{V}\left(I_{n-2}, I_{n-3}, G_{n-2}^{\prime \prime}\right) \subset \mathbb{P}^{2 n-5}\left(\right.$ no $\left.B_{n-1}, A_{n-2}, B_{n-2}, A_{n-1}\right)$. The sequence (4.39) simplifies to

$$
\begin{aligned}
\longrightarrow & H_{\text {prim }}^{r-7}(S)(-1) \longrightarrow H_{c}^{r-5}\left(U_{2}\right) \longrightarrow \\
& H_{\text {prim }}^{r-5}\left(\mathcal{V}\left(I_{n-2}, A_{n-3} I_{n-3}, G_{n-2}\right)\right) \longrightarrow H_{\text {prim }}^{r-7}(S)(-1) \longrightarrow
\end{aligned}
$$


Applying the functors $\operatorname{gr}_{i}^{W}$ to the sequence, by (4.34), (4.36), (4.38) and (4.40), we get

$$
\operatorname{gr}_{i}^{W} H_{\operatorname{prim}}^{r-4}(V) \cong \operatorname{gr}_{i}^{W} H_{\mathrm{c}}^{r-5}\left(U_{2}\right) \quad \text { for } i=0,1 .
$$

Now, the scheme $U_{2}$ is defined by the system

$$
\left\{\begin{array} { r l } 
{ I _ { n - 2 } = G _ { n - 2 } } & { = 0 , } \\
{ A _ { n - 3 } I _ { n - 3 } } & { = 0 , } \\
{ I _ { n - 3 } } & { \neq 0 , }
\end{array} \Leftrightarrow \left\{\begin{array}{rl}
G_{n-2}=I_{n-2} & =0, \\
A_{n-3} & =0, \\
I_{n-3} & \neq 0 .
\end{array}\right.\right.
$$

Eliminating $A_{n-3}$, which is zero on $U_{2}$, we get an isomorphism

$$
U_{2} \cong U_{2}^{\prime}
$$

with $U_{2}^{\prime}:=\mathcal{V}\left(I_{n-2}^{\prime}, G_{n-2}^{\prime}\right) \backslash \mathcal{V}\left(I_{n-2}^{\prime}, G_{n-2}^{\prime}, I_{n-3}\right) \subset \mathbb{P}^{2 n-5} \quad$ (no $B_{n-1}, A_{n-2}$, $\left.B_{n-2}, A_{n-3}\right)$, where primes mean that we set $A_{n-3}=0$ in the polynomials, namely in $C_{n-3}$. Now we write

$$
C_{n-3}^{\prime}=A_{n-1} \pm A_{n-4},
$$

with "+" only when $a_{n-4 n-4}=B_{n-4}$ in the matrix.

By Corollary 1.3, it follows that $U_{2}^{\prime}$ is defined by the system

$$
\left\{\begin{aligned}
C_{n-3}^{\prime} I_{n-3}-A_{n-4}^{2} I_{n-4} & =0, \\
L_{n-2} & =0, \\
I_{n-3} & \neq 0,
\end{aligned}\right.
$$

with

$$
\begin{aligned}
L_{n-2} & :=A_{n-1} I_{n-3}+\sum_{s}(-1)^{s+n-1} A_{v(s)} I_{n-2}(s, n-3) \\
& =A_{n-1} I_{n-3}+\sum_{s}(-1)^{s+n-1} A_{v(s)} I_{s} \prod_{k=s}^{n-4} A_{k} .
\end{aligned}
$$

The sum goes over all $s=n_{i}+j-1<n-3, i \not \equiv t \bmod 2,1 \leq i \leq t, 1 \leq$ $j \leq l_{i}$, so over all $s<n-3$ such that $a_{s s}=C_{s}$. It is convenient to use the 
recurrence formula

$$
L_{s+1}=\left\{\begin{aligned}
A_{v(s)} I_{s}-A_{s-1} L_{s}, & a_{s+1 s+1}=C_{s+1} \\
-A_{s-1} L_{s}, & a_{s+1 s+1}=B_{s+1}
\end{aligned}\right.
$$

We can express $A_{n-1}$ from the second equation of the system (4.45) and $C_{2}$ from the first one.

$$
\left\{\begin{array}{r}
A_{n-1} \pm A_{n-4}=C_{n-3}^{\prime}=A_{n-4}^{2} I_{n-4} / I_{n-3} \\
A_{n-1}=A_{n-4} L_{n-3} / I_{n-3} \\
I_{n-3} \neq 0 .
\end{array}\right.
$$

These two expressions for $A_{n-1}$ must be equal on $U_{2}^{\prime}$. We introduce the polynomials $N_{s}$ defined by $A_{s-1} N_{s}= \pm A_{s-1} I_{s}+A_{s-1}^{2} I_{s-1}-A_{s-1} L_{s}$. Sometimes we write $N_{s}^{-}$and $N_{s}^{+}$to indicate the sign taken in the expression on the right. The natural projection from the point where all the variables but $A_{n-1}$ are zero induces an isomorphism

$$
U_{2}^{\prime} \cong U_{3}:=\mathcal{V}\left(A_{n-4} N_{n-3}\right) \backslash \mathcal{V}\left(A_{n-4} N_{n-3}, I_{n-3}\right)
$$

with $U_{3} \subset \mathbb{P}^{2 n-6}$ (no $\left.B_{n-1}, A_{n-2}, B_{n-2}, A_{n-3}, A_{n-1}\right)$. By (4.41), and (4.43),

$$
\operatorname{gr}_{i}^{W} H_{\text {prim }}^{r-4}(V) \cong \operatorname{gr}_{i}^{W} H_{\mathrm{c}}^{r-5}\left(U_{3}\right) \text { for } i=0,1
$$

We have two possibilities : $a_{n-4 n-4}=C_{n-4}$ or $a_{n-4 n-4}=B_{n-4}$. When the latter holds, go to Step 4 with $N_{n-3}=N_{n-3}^{-}$; do the next step with $N_{n-3}=$ $N_{n-3}^{+}$otherwise.

Step 3. Suppose that the entry $a_{s s}$ of $M_{\mathrm{GZZ}}$ is $C_{s}$ and $a_{s+1 s+1}=C_{s+1}$. In other words, $n_{i} \leq s \leq n_{i}+l_{i}-2$ for some $i \not \equiv t \bmod 2$. This corresponds to the case $s=n-4$ if we had come from Step 2. One has

$$
C_{s}=A_{v}-A_{s} \pm A_{s-1}
$$

with "+" only when $a_{s-1 s-1}=B_{s}$. We work in $\mathbb{P}^{N}\left(\right.$ no $\left.\mathrm{DV}_{s}\right)$ for $N=2 n-$ $1-2(n-1-s-1)-1=2 s+2$, and the dropped variables $\left(\mathrm{DV}_{s}\right)$ are all the variables in $I_{n-1-s}^{s+1}$ but $A_{s}$. We are going to compute $H_{\mathrm{c}}^{q}(U)$ for $q \geq 2 s+1$ 
and $U$ defined by

$$
U:=\mathcal{V}\left(A_{s} N_{s+1}\right) \backslash \mathcal{V}\left(A_{s} N_{s+1}, I_{s+1}\right)
$$

where $N_{s+1}=I_{s+1}+A_{s} I_{s}-L_{s+1}$. Let $T:=\mathcal{V}\left(A_{s} N_{s+1}\right), Y:=\mathcal{V}\left(A_{s} N_{s+1}\right.$, $\left.I_{s+1}\right)$ in $\mathbb{P}^{2 s+2}\left(\right.$ no $\left.\mathrm{DV}_{s}\right)$. One has an exact sequence

$$
\rightarrow H_{\mathrm{prim}}^{q-1}(T) \rightarrow H_{\mathrm{prim}}^{q-1}(Y) \rightarrow H_{\mathrm{c}}^{q}(U) \rightarrow H_{\mathrm{prim}}^{q}(T) \rightarrow
$$

Using (4.51), we rewrite

$$
\begin{aligned}
N_{s+1} & =\left(A_{v}-A_{s} \pm A_{s-1}\right) I_{s}-A_{s-1}^{2} I_{s-1}+A_{s} I_{s}-A_{v} I_{s}+A_{s-1} L_{s} \\
& =-A_{s-1}\left( \pm I_{s}+A_{s-1} I_{s-1}-L_{s}\right)=-A_{s-1} N_{s}
\end{aligned}
$$

and see that $N_{s+1}$ is actually independent of $A_{v}$ and $A_{s}$. After applying of $h . i$. and $\operatorname{gr}_{*}^{W}$, the sequence (4.53) yields the isomorphism

$$
\operatorname{gr}_{i}^{W} H_{\mathrm{c}}^{q}(U) \cong \operatorname{gr}_{i}^{W} H_{\mathrm{prim}}^{q-1}(Y), \quad i=0,1 .
$$

Define $\hat{Y}_{1}:=Y \cap \mathcal{V}\left(I_{s}\right)=\mathcal{V}\left(A_{s} N_{s+1}, I_{s}, A_{s-1} I_{s-1}\right)$. The polynomial $N_{s+1}$ is independent of $A_{v}$ by (4.54). Using $h$.i. for $\hat{Y}_{1}$, we come to an exact sequence

$$
\begin{array}{r}
H_{\mathrm{prim}}^{q-4}\left(Y_{1}\right)(-1) \rightarrow H_{\mathrm{c}}^{q-1}\left(Y \backslash \hat{Y}_{1}\right) \rightarrow \\
H_{\mathrm{prim}}^{q-1}(Y) \rightarrow H_{\mathrm{prim}}^{q-3}\left(Y_{1}\right)(-1) \rightarrow
\end{array}
$$

with $Y_{1} \subset \mathbb{P}^{2 s+1}\left(\right.$ no $\left.\mathrm{DV}_{s}, A_{v}\right)$ defined by the same polynomials. The scheme $Y \backslash \hat{Y}_{1}$ is given by the system

$$
\left\{\begin{aligned}
A_{s} A_{s-1} N_{s} & =0 \\
C_{s} I_{s}-A_{s-1}^{2} I_{s-1} & =0 \\
I_{s} & \neq 0 .
\end{aligned}\right.
$$

By (4.51), we express $A_{v}$ from the second equation. Projecting from the point where all the variables but $A_{v}$ are zero, we get isomorphisms

$$
Y \backslash \hat{Y}_{1} \cong R \quad \text { and } \quad H_{\mathrm{c}}^{q-1}\left(Y \backslash \hat{Y}_{1}\right) \cong H_{\mathrm{c}}^{q-1}(R)
$$

where $R:=\mathcal{V}\left(A_{s} A_{s-1} N_{s}\right) \backslash \mathcal{V}\left(A_{s} A_{s-1} N_{s}, I_{s}\right) \subset \mathbb{P}^{2 s+1}\left(\right.$ no $\left.\mathrm{DV}_{s}, A_{v}\right)$. Define $R_{1}:=\mathcal{V}\left(A_{s-1} N_{s}\right) \backslash \mathcal{V}\left(A_{s-1} N_{s}, I_{s}\right)$ and $R_{2}:=\mathcal{V}\left(A_{s}\right) \backslash \mathcal{V}\left(A_{s}, I_{s}\right)$. One has the 
Mayer-Vietoris sequence

$$
\begin{aligned}
\longrightarrow & H_{\mathrm{c}}^{q-2}\left(R_{1}\right) \oplus H_{\mathrm{c}}^{q-2}\left(R_{2}\right) \longrightarrow H_{\mathrm{c}}^{2 s-1}\left(R_{3}\right) \longrightarrow \\
& H_{\mathrm{c}}^{q-1}(R) \longrightarrow H_{\mathrm{c}}^{q-1}\left(R_{1}\right) \oplus H_{\mathrm{c}}^{q-1}\left(R_{2}\right) \longrightarrow,
\end{aligned}
$$

with $R_{3}:=R_{1} \cap R_{2}$. The defining polynomials of $R_{1}$ and $R_{2}$ are independent of $A_{s}$ and $A_{m}$, respectively. Applying h.i. to them, and applying functors $\mathrm{gr}_{i}^{W}$ to the sequence above, we get isomorphisms

$$
\operatorname{gr}_{i}^{W} H_{\mathrm{c}}^{q-1}(R) \cong \operatorname{gr}_{i}^{W} H_{\mathrm{c}}^{q-2}\left(R_{3}\right), \quad i=0,1 .
$$

Now, $R_{3}:=\mathcal{V}\left(A_{s}, A_{s-1} N_{s}\right) \backslash \mathcal{V}\left(A_{s}, A_{s-1} N_{s}, I_{s}\right) \subset \mathbb{P}^{2 s+1}\left(\right.$ no $\left.\mathrm{DV}_{s}, A_{v}\right)$. Projecting from the point where all the variables but $A_{s}$ are zero, we get isomorphisms

$$
R_{3} \cong U^{\prime} \quad \text { and } \quad H_{c}^{q-2}\left(R_{3}\right) \cong H_{c}^{q-2}\left(U^{\prime}\right)
$$

for $U^{\prime}=\mathcal{V}\left(A_{s-1} N_{s}\right) \backslash \mathcal{V}\left(A_{s-1} N_{s}, I_{s}\right) \subset \mathbb{P}^{2 s}\left(\right.$ no $\left.\mathrm{DV}_{s}, A_{v}, A_{s}\right)$. Collecting (4.55), (4.56), (4.58), (4.60) and (4.61) together, we obtain

$$
\operatorname{gr}_{i}^{W} H_{\mathrm{c}}^{q}(U) \cong \operatorname{gr}_{i}^{W} H_{\mathrm{c}}^{q-2}\left(U^{\prime}\right), \quad i=0,1,
$$

where $U$ is defined by (4.52) and $q \geq 2 s+1$.

If $s=1$, go to the last step.

When we come to Step 3 with some $s, n_{i} \leq s \leq n_{i}+l_{i}-2, i \not \equiv t \bmod 2$, we must apply this step $s-n_{i}-1$ times with $N_{s}=N_{s}^{+}$and then one more time with $N_{s}=N_{s}^{-}$. After this, we are in a new situation.

Step 4. Suppose that the entry $a_{s s}$ of $M_{\mathrm{GZZ}}$ is $B_{s}$ and $a_{s+1 s+1}=C_{s+1}$. This means that $s=n_{i}-1$ for some $i \not \equiv t \bmod 2$. Denote by $\mathrm{DV}_{s}$ the dropped variables that are all the variables appearing in $I_{n-1-s}^{s+1}$ but $A_{s}$. Again, we want to compute $H_{\mathrm{c}}^{q}(U)$ for $q \geq 2 s+1$ and $U \subset \mathbb{P}^{2 s+2}$ defined by

$$
U:=\mathcal{V}\left(A_{s} N_{s+1}\right) \backslash \mathcal{V}\left(A_{s} N_{s+1}, I_{s+1}\right),
$$

where $N_{s+1}=-I_{s+1}+A_{s} I_{s}-L_{s+1}$. Define $U_{1}:=\mathcal{V}\left(A_{s}\right) \backslash \mathcal{V}\left(A_{s}, I_{s+1}\right), U_{2}:=$ $\mathcal{V}\left(N_{s+1}\right) \backslash \mathcal{V}\left(N_{s+1}, I_{s+1}\right)$. This covering gives us an exact sequence

$$
\begin{gathered}
\longrightarrow H_{\mathrm{c}}^{q-1}\left(U_{1}\right) \oplus H_{\mathrm{c}}^{q-1}\left(U_{1}\right) \longrightarrow H_{\mathrm{c}}^{2 s}\left(U_{3}\right) \longrightarrow \\
H_{\mathrm{c}}^{q}(U) \longrightarrow H_{\mathrm{c}}^{q}\left(U_{1}\right) \oplus H_{\mathrm{c}}^{q}\left(U_{1}\right) \longrightarrow,
\end{gathered}
$$


where $U_{3}:=U_{1} \cap U_{2}$. The polynomials in the definition of $U_{1}$ do not depend on $A_{m}$. Moreover, we can rewrite

$$
\begin{aligned}
N_{s+1}= & -I_{s+1}+A_{s} I_{s}-L_{s+1}=-B_{s} I_{s}+A_{s-1}^{2} I_{s-1}+A_{s} I_{s} \\
& +A_{s-1} L_{s}=\left(A_{s}-B_{s}\right) I_{s}+A_{s-1}^{2} I_{s-1}+A_{s-1} L_{s}
\end{aligned}
$$

and see that $N_{s+1}$ depends neither on $B_{s}$ nor on $A_{s}$ but on the difference $A_{s}-B_{s}$. After the change of variables $B_{s}:=A_{s}-B_{s}$, the polynomial $N_{s+1}$ becomes independent of $A_{s}$. Applying h.i. to $U_{1}$ and $U_{2}$, and then applying the functors $\operatorname{gr}_{i}^{W}$ to the sequence (4.64), we get

$$
\operatorname{gr}_{i}^{W} H_{\mathrm{c}}^{q}(U) \cong \operatorname{gr}_{i}^{W} H_{\mathrm{c}}^{q-1}\left(U_{3}\right), \quad i=0,1
$$

Now, $U_{3} \subset \mathbb{P}^{2 s+2}\left(\right.$ no $\left.\mathrm{DV}_{s}\right)$ is given by the system

$$
\left\{\begin{array} { r l } 
{ A _ { s } } & { = 0 , } \\
{ N _ { s + 1 } } & { = 0 , } \\
{ I _ { s + 1 } } & { \neq 0 , }
\end{array} \Leftrightarrow \left\{\begin{array}{r}
A_{s}=0 \\
I_{s+1}+L_{s+1}=0 \\
I_{s+1} \neq 0 .
\end{array}\right.\right.
$$

We eliminate the variable $A_{s}$ and consider an open $U_{4} \subset \mathbb{P}^{2 s+1}\left(\right.$ no $\left.\mathrm{DV}_{s}, A_{s}\right)$ defined by the last two conditions, then

$$
H_{\mathrm{c}}^{q-1}\left(U_{3}\right) \cong H_{\mathrm{c}}^{q-1}\left(U_{4}\right)
$$

Define $T:=\mathcal{V}\left(I_{s+1}+L_{s+1}\right), Y:=\mathcal{V}\left(I_{s+1}+L_{s+1}, I_{s+1}\right)$ in $\mathbb{P}^{2 s+1}\left(\right.$ no $\mathrm{DV}_{s}$, $\left.A_{s}\right)$. We can write an exact sequence

$$
\longrightarrow H_{\mathrm{prim}}^{q-2}(T) \longrightarrow H_{\mathrm{prim}}^{q-2}(Y) \longrightarrow H_{\mathrm{c}}^{q-1}\left(U_{4}\right) \longrightarrow H_{\mathrm{prim}}^{q-1}(T) \longrightarrow
$$

Similar to (4.65), one has $I_{s+1}+L_{s+1}=B_{s} I_{s}-A_{s-1}^{2} I_{s-1}-A_{s-1} L_{s}$. Let $T_{1}:=T \cap \mathcal{V}\left(I_{s}\right)=\mathcal{V}\left(I_{s}, A_{s-1}^{2} I_{s-1}+A_{s-1} L_{s}\right) \subset \mathbb{P}^{2 s+1}\left(\right.$ no $\left.\mathrm{DV}_{s}, A_{s}\right)$. For $p=$ $q-1$ and $p=q-2$ we write an exact sequence

$$
\longrightarrow H_{\mathrm{c}}^{p}\left(T \backslash T_{1}\right) \longrightarrow H_{\mathrm{prim}}^{p}(T) \longrightarrow H_{\mathrm{prim}}^{p}\left(T_{1}\right) \longrightarrow
$$

On $T \backslash T_{1}$ we can express $B_{s}$ and get an isomorphism $T \backslash T_{1} \cong \mathbb{P}^{2 s} \backslash \mathcal{V}\left(I_{s}\right)$ with $\mathbb{P}^{2 s}\left(\right.$ no $\left.\mathrm{DV}_{s}, A_{s}, B_{s}\right)$. The polynomial $I_{s}$ does not depend on $A_{m}$. Furthermore, the defining polynomials of $T_{1}$ are independent of $B_{s}$. We apply $h . i$. 
to $T \backslash T_{1}$ and $T_{1}$, and apply $\operatorname{gr}_{i}^{W}$ to the sequence (4.70). We get

$$
\operatorname{gr}_{i}^{W} H_{\text {prim }}^{p}(T)=0, \quad i=0,1, \quad p=q-1, q-2 .
$$

We return to $Y=\mathcal{V}\left(L_{s+1}, I_{s+1}\right)=\mathcal{V}\left(A_{s-1} L_{s}, B_{s} I_{s}-A_{s-1}^{2} I_{s-1}\right)$. One can write an exact sequence

$$
\rightarrow H_{\mathrm{prim}}^{q-3}\left(Y_{1}\right) \rightarrow H_{\mathrm{c}}^{q-2}\left(Y \backslash Y_{1}\right) \rightarrow H_{\mathrm{prim}}^{q-2}(Y) \rightarrow H_{\mathrm{prim}}^{q-2}\left(Y_{1}\right) \rightarrow
$$

where $Y_{1}:=Y \cap \mathcal{V}\left(I_{s}\right)=\mathcal{V}\left(I_{s}, A_{s-1} L_{s-1}, A_{s-1} I_{s-1}\right) \subset \mathbb{P}^{2 s+1}\left(\right.$ no $\left.\mathrm{DV}_{s}, A_{s}\right)$. The last three polynomials are independent of $B_{s}$. After applying h.i. and taking $\operatorname{gr}_{i}^{W}$, the sequence (4.72) implies

$$
\operatorname{gr}_{i}^{W} H_{\mathrm{prim}}^{q-2}(Y) \cong \operatorname{gr}_{i}^{W} H_{\mathrm{c}}^{q-2}\left(Y \backslash Y_{1}\right), \quad i=0,1 .
$$

Now, $Y \backslash Y_{1} \subset \mathbb{P}^{2 s+1}$ (no $\left.\mathrm{DV}_{s}, A_{s}\right)$ is defined by the system

$$
\left\{\begin{aligned}
A_{s-1} L_{s} & =0 \\
B_{s} I_{s}-A_{s-1}^{2} I_{s-1} & =0 \\
I_{s} & \neq 0
\end{aligned}\right.
$$

We can express $B_{s}$ from the second equation, and we get an isomorphism $Y \backslash \hat{Y}_{1} \cong U^{\prime}$, where

$$
U^{\prime}:=\mathcal{V}\left(A_{s-1} L_{s}\right) \backslash \mathcal{V}\left(A_{s-1} L_{s}, I_{s}\right) \subset \mathbb{P}^{2 s}\left(\text { no } \mathrm{DV}_{s}, A_{s}, B_{s}\right)
$$

Finally, combining (4.66), (4.68), (4.69), (4.71) and (4.73), we get

$$
\operatorname{gr}_{i}^{W} H_{\mathrm{c}}^{q}(U) \cong \operatorname{gr}_{i}^{W} H^{q-2}(Y) \cong \operatorname{gr}_{i}^{W} H_{\mathrm{c}}^{q-2}\left(U^{\prime}\right), \quad i=0,1
$$

for $U$ and $U^{\prime}$ defined by (4.63) and (4.75), respectively, and $q \geq 2 s+1$.

Now, if $s=1$, go to the last step. If $a_{s-1 s-1}=C_{s-1}$, we go to Step 6 . Otherwise do the next step.

Step 5. Consider an entry $a_{s s}=B_{s}$ of $M_{\mathrm{GZZ}}$ such that $a_{s+1 s+1}=B_{s+1}$. With other words, $s$ satisfies the condition $n_{i} \leq s \leq n_{i}+l_{i+1}-2$ for some $i \equiv$ $t \bmod 2$. Let $U \subset \mathbb{P}^{2 s+2}\left(\right.$ no $\left.\mathrm{DV}_{s}\right)$ be defined by

$$
U:=\mathcal{V}\left(A_{s} L_{s+1}\right) \backslash \mathcal{V}\left(A_{s} L_{s+1}, I_{s+1}\right),
$$

and denote by $\mathrm{DV}_{s}$ all the variables appearing in $I_{n-1-s}^{s+1}$. As usual, we try to compute $H_{\mathrm{c}}^{q}(U)$ for $q \geq 2 s+1$. Define $U_{1}:=\mathcal{V}\left(A_{s}\right) \backslash \mathcal{V}\left(A_{s}, I_{s+1}\right)$, 
$U_{2}:=\mathcal{V}\left(L_{s+1}\right) \backslash \mathcal{V}\left(L_{s+1}, I_{s+1}\right)$ in $\mathbb{P}^{2 s+2}$. One can write an exact sequence

$$
\begin{gathered}
\longrightarrow H_{\mathrm{c}}^{q-1}\left(U_{1}\right) \oplus H_{\mathrm{c}}^{q-1}\left(U_{2}\right) \longrightarrow H_{\mathrm{c}}^{q-1}\left(U_{3}\right) \longrightarrow \\
H_{\mathrm{c}}^{q}(U) \longrightarrow H_{\mathrm{c}}^{q}\left(U_{1}\right) \oplus H_{\mathrm{c}}^{q}\left(U_{2}\right) \longrightarrow,
\end{gathered}
$$

where $U_{3}:=U_{1} \cap U_{2}$. The defining polynomials of $U_{1}$ do not depend on $A_{m}$, thus Theorem B $(N=2 s+2, k=1, t=1)$ implies $H_{\mathrm{c}}^{i}\left(U_{1}\right)=0$ for $i<$ $2 s+2$. Since $L_{s+1}=-A_{s-1} L_{s}$ and $I_{s+1}$ are independent of $A_{s}$, and $I_{s+1}$ is also independent of $A_{m}$, we can apply h.i. to $U_{1}$ and $U_{2}$. After applying $\operatorname{gr}_{i}^{W}$ to the sequence (4.78), one gets

$$
\operatorname{gr}_{i}^{W} H_{\mathrm{c}}^{q}(U) \cong \operatorname{gr}_{i}^{W} H_{\mathrm{c}}^{q-1}\left(U_{3}\right), \quad i=0,1 .
$$

We eliminate $A_{s}$, which is zero along $U_{3}$, and get an isomorphism

$$
U_{3} \cong U_{4}:=\mathcal{V}\left(L_{s+1}\right) \backslash \mathcal{V}\left(L_{s+1}, I_{s+1}\right),
$$

with $U_{4} \subset \mathbb{P}^{2 s+1}$ (no $\left.\mathrm{DV}_{s}, A_{s}\right)$. Defining $T:=\mathcal{V}\left(L_{s+1}\right), Y:=\mathcal{V}\left(L_{s+1}, I_{s+1}\right)$ in $\mathbb{P}^{2 s+1}$, we get an exact sequence

$$
\rightarrow H_{\mathrm{prim}}^{q-2}(T) \rightarrow H_{\mathrm{prim}}^{q-2}(Y) \rightarrow H_{\mathrm{c}}^{q-1}\left(U_{4}\right) \rightarrow H_{\mathrm{prim}}^{q-1}(T) \rightarrow .
$$

Since $L_{s+1}$ is independent of $B_{s}$, h.i. applied to $T$ implies

$$
\operatorname{gr}_{i}^{W} H_{\mathrm{c}}^{q-1}\left(U_{4}\right) \cong \operatorname{gr}_{i}^{W} H_{\operatorname{prim}}^{q-2}(Y), \quad i=0,1 .
$$

Let $Y_{1}:=Y \cap \mathcal{V}\left(I_{s}\right)=\mathcal{V}\left(L_{s}, I_{s+1}, I_{s}\right)$. One has an exact sequence

$$
\rightarrow H_{\mathrm{prim}}^{q-3}\left(Y_{1}\right) \rightarrow H_{\mathrm{c}}^{q-2}\left(Y \backslash Y_{1}\right) \rightarrow H_{\mathrm{prim}}^{q-2}(Y) \rightarrow H_{\mathrm{prim}}^{q-2}\left(Y_{1}\right) \rightarrow .
$$

Since $\quad Y_{1}=\mathcal{V}\left(L_{s+1}, B_{s} I_{s}-A_{s-1}^{2} I_{s-1}, I_{s}\right)=\mathcal{V}\left(A_{s-1} L_{s}, I_{s}, A_{s-1} I_{s-1}\right)$, the defining polynomials forget $B_{s}$. Applying h.i. to $Y_{1}$, and $\operatorname{gr}_{i}^{W}$ to the sequence (4.83), one gets

$$
\operatorname{gr}_{i}^{W} H_{\operatorname{prim}}^{q-2}(Y) \cong \operatorname{gr}_{i}^{W} H_{\mathrm{c}}^{q-2}\left(Y \backslash Y_{1}\right), \quad i=0,1 .
$$

The open subscheme $Y \backslash Y_{1} \subset Y$ is given by the system

$$
\left\{\begin{aligned}
A_{s-1} L_{s-1} & =0 \\
B_{s} I_{s}-A_{s-1}^{2} I_{s-1} & =0 \\
I_{s} & \neq 0
\end{aligned}\right.
$$


Expressing $B_{s}$ from the second equation and projecting from the point where all the variables but $B_{s}$ are zero, we get an isomorphism $Y \backslash Y_{1} \cong U^{\prime}$, where

$$
U^{\prime}:=\mathcal{V}\left(A_{s-1} L_{s}\right) \backslash \mathcal{V}\left(A_{s-1} L_{s}, I_{s}\right) \subset \mathbb{P}^{2 s}
$$

Collecting now $(4.79),(4.80),(4.82)$ and (4.84) together, we obtain

$$
\operatorname{gr}_{i}^{W} H_{\mathrm{c}}^{q}(U) \cong \operatorname{gr}_{i}^{W} H_{\mathrm{c}}^{q-2}\left(U^{\prime}\right), \quad i=0,1
$$

for $U$ and $U^{\prime}$ defined by (4.77) and (4.86), respectively, and $q \geq 2 s+1$.

If $s=1$, go to the last step.

After repeating a suitable number of times Step 5, we come to the following situation.

Step 6. Suppose that the entry $a_{s s}$ of the matrix $M_{\mathrm{GZZ}}$ is $C_{s}$ and $a_{s+1 s+1}=B_{s+1}$. This happens when $s=n_{i}-1$ for some $i \equiv t \bmod 2$. For $C_{s}$, we have

$$
C_{s}=A_{v}+A_{s} \pm A_{s-1}
$$

with "+" only when $l_{i}=1$. Let $U \subset \mathbb{P}^{2 s+2}\left(\right.$ no $\left.\mathrm{DV}_{s}\right)$ be defined by

$$
U:=\mathcal{V}\left(A_{s} L_{s+1}\right) \backslash \mathcal{V}\left(A_{s} L_{s+1}, I_{s+1}\right)
$$

and denote by $\mathrm{DV}_{s}$ all the variables appearing in $I_{n-1-s}^{s+1}$. As in the previous case, we define $U_{1}:=\mathcal{V}\left(A_{s}\right) \backslash \mathcal{V}\left(A_{s}, I_{s+1}\right), U_{2}:=\mathcal{V}\left(L_{s+1}\right) \backslash \mathcal{V}\left(L_{s+1}, I_{s+1}\right)$ and write an exact sequence

$$
\begin{aligned}
\longrightarrow & H_{\mathrm{c}}^{q-1}\left(U_{1}\right) \oplus H_{\mathrm{c}}^{q-1}\left(U_{2}\right) \longrightarrow H_{\mathrm{c}}^{q-1}\left(U_{3}\right) \longrightarrow \\
& H_{\mathrm{c}}^{q}(U) \longrightarrow H_{\mathrm{c}}^{q}\left(U_{1}\right) \oplus H_{\mathrm{c}}^{q}\left(U_{2}\right) \longrightarrow,
\end{aligned}
$$

where $U_{3}:=U_{1} \cap U_{2}$ and $q \geq 2 s+1$. For this step, we have

$$
\begin{gathered}
L_{s+1}=A_{v} I_{s}-A_{s-1} L_{s}, \\
I_{s+1}=C_{s} I_{s}-A_{s-1}^{2} I_{s-1},
\end{gathered}
$$

so the polynomials defining $U_{1}$ and $U_{2}$ are independent of $A_{m}$ and $A_{s}$, respectively. We apply $h . i$. to $U_{1}$ and $U_{2}$, and the sequence (4.90) yields

$$
\operatorname{gr}_{i}^{W} H_{\mathrm{c}}^{q}(U) \cong \operatorname{gr}_{i}^{W} H_{\mathrm{c}}^{q-1}\left(U_{3}\right), \quad i=0,1
$$


Eliminating $A_{s}$, which is zero on $U_{3}$, we get an isomorphism

$$
U_{3} \cong U_{4}:=\mathcal{V}\left(L_{s+1}\right) \backslash \mathcal{V}\left(L_{s+1}, I_{s+1}\right)
$$

with $U_{4} \subset \mathbb{P}^{2 s+1}$ (no $\left.\mathrm{DV}_{s}, A_{s}\right)$. Denoting by $I_{s+1}^{\prime}$ the polynomial $I_{s+1}$ after setting $A_{s}=0$, we define $T:=\mathcal{V}\left(L_{s+1}\right)$ and $Y:=\mathcal{V}\left(L_{s+1}, I_{s+1}^{\prime}\right)$ in $\mathbb{P}^{2 s+1}$. One gets an exact sequence

$$
\rightarrow H_{\mathrm{prim}}^{q-2}(T) \rightarrow H_{\mathrm{prim}}^{q-2}(Y) \rightarrow H_{\mathrm{c}}^{q-1}\left(U_{4}\right) \rightarrow H_{\mathrm{prim}}^{q-1}(T) \rightarrow
$$

Motivated by (4.91), we define $T_{1}:=T \cap \mathcal{V}\left(I_{s}\right)=\mathcal{V}\left(I_{s}, A_{s-1} L_{s}\right)$. One can write an exact sequence

$$
\longrightarrow H_{\mathrm{c}}^{p}\left(T \backslash T_{1}\right) \longrightarrow H_{\mathrm{prim}}^{p}(T) \longrightarrow H_{\mathrm{prim}}^{p}\left(T_{1}\right) \longrightarrow,
$$

where $p=q-1$ or $p=q-2$. On $T \backslash T_{1}$ we can express $A_{v}$ from the equation $L_{s+1}=0$. Projecting from the point where all the variables but $A_{v}$ are zero, we get an isomorphism $T \backslash T_{1} \cong \mathbb{P}^{2 s} \backslash \mathcal{V}\left(I_{s}\right)$. The polynomial $I_{s}$ does not depend on $A_{m}$. Furthermore, the polynomials defining $T_{1}$ are independent of $A_{v}$. Applying h.i. to $T_{1}$ and $T \backslash T_{1}$, and then applying the functors $\operatorname{gr}_{i}^{W}$ to the sequence $(4.95)$, we obtain

$$
\operatorname{gr}_{0}^{W} H_{\text {prim }}^{p}(T)=\operatorname{gr}_{1}^{W} H_{\text {prim }}^{p}(T)=0, \quad p=q-1, q-2 .
$$

Let $Y_{1}:=\mathcal{V}\left(L_{s+1}, I_{s+1}^{\prime}, I_{s}\right)=\mathcal{V}\left(A_{s-1} L_{s}, A_{s-1} I_{s-1}, I_{s}\right) \subset Y$. One has an exact sequence

$$
\rightarrow H_{\mathrm{prim}}^{q-3}\left(Y_{1}\right) \rightarrow H_{\mathrm{c}}^{q-2}\left(Y \backslash Y_{1}\right) \rightarrow H_{\mathrm{prim}}^{q-2}(Y) \rightarrow H_{\mathrm{prim}}^{q-2}\left(Y_{1}\right) \rightarrow
$$

The defining polynomials of $Y_{1}$ do not depend on $A_{v}$. Using h.i. and $\operatorname{gr}_{i}^{W}$, we get

$$
\operatorname{gr}_{i}^{W} H_{\operatorname{prim}}^{q-2}(Y) \cong \operatorname{gr}_{i}^{W} H_{c}^{q-2}\left(Y \backslash Y_{1}\right), \quad i=0,1
$$

The open subscheme $Y \backslash \hat{Y}_{1} \subset Y$ is defined by the system

$$
\left\{\begin{array} { r l } 
{ L _ { s + 1 } } & { = 0 , } \\
{ I _ { s + 1 } ^ { \prime } } & { = 0 , } \\
{ I _ { s } } & { \neq 0 , }
\end{array} \Leftrightarrow \left\{\begin{array}{r}
A_{v} I_{s}-A_{s-1} L_{s}=0, \\
\left(A_{v} \pm A_{s-1}\right) I_{s}-A_{s-1}^{2} I_{s-1}=0, \\
I_{s} \neq 0 .
\end{array}\right.\right.
$$

We can express $A_{v}$ from the first and second equations and there expressions must be equal. So we define $N_{s}:= \pm I_{s}+A_{s-1} I_{s-1}-L_{s}$ with "_" only when 
$l_{i}=1$. The expression for $A_{v}$ and the natural projection from the point where all the variables but $A_{v}$ are zero yield an isomorphism $Y \backslash \hat{Y}_{1} \cong U^{\prime}$, where

$$
U^{\prime}:=\mathcal{V}\left(A_{s-1} N_{s}\right) \backslash \mathcal{V}\left(A_{s-1} N_{s}, I_{s}\right) \subset \mathbb{P}^{2 s}
$$

Collecting (4.92), (4.93), (4.96) and (4.98) together, one gets

$$
\operatorname{gr}_{i}^{W} H_{\mathrm{c}}^{q}(U) \cong \operatorname{gr}_{i}^{W} H_{\mathrm{c}}^{q-2}\left(U^{\prime}\right), \quad i=0,1
$$

with $U$ and $U^{\prime}$ defined by (4.89) and (4.100), respectively.

If $s=1$, go to the last step. If $a_{s-1 s-1}=B_{s-1}$, return to Step 4 with $N_{s}=N_{s}^{-}$; return to Step 3 with $N_{s}=N_{s}^{+}$otherwise.

The last step. Recall that $l_{1}>1$. In the case $t \equiv 0 \bmod 2$, we have come from Step 4 or Step 5. The matrix looks like

$$
\mathcal{M}_{\mathrm{GZZ}}=\left(\begin{array}{cccc}
B_{0} & A_{0} & \vdots & A_{m} \\
A_{0} & B_{1} & \vdots & 0 \\
\ldots & \ldots & \ddots & \vdots \\
A_{m} & 0 & \ldots & \ddots
\end{array}\right) .
$$

We are interested in $H^{q}(U)$ for $q \geq 1$, where $U:=\mathcal{V}\left(A_{0} L_{1}\right) \backslash \mathcal{V}\left(A_{0} L_{1}, I_{1}\right)=$ $\mathcal{V}\left(A_{0} A_{m}\right) \backslash \mathcal{V}\left(A_{0} A_{m}, B_{0}\right) \subset \mathbb{P}^{2}\left(A_{0}: A_{m}: B_{0}\right)$. One has an exact sequence

$$
\begin{aligned}
\longrightarrow & H_{\text {prim }}^{0}\left(\mathcal{V}\left(A_{0} A_{m}\right)\right) \longrightarrow H_{\text {prim }}^{0}\left(\mathcal{V}\left(A_{0} A_{m}, B_{0}\right)\right) \longrightarrow H_{c}^{1}(U) \longrightarrow \\
& H^{1}\left(\mathcal{V}\left(A_{0} A_{m}\right)\right) \longrightarrow H^{1}\left(\mathcal{V}\left(A_{0} A_{m}, B_{0}\right)\right) \longrightarrow H_{c}^{2}(U) \longrightarrow \\
& H^{2}\left(\mathcal{V}\left(A_{0} A_{m}\right)\right) \longrightarrow H^{2}\left(\mathcal{V}\left(A_{0} A_{m}, B_{0}\right)\right) \longrightarrow .
\end{aligned}
$$

The varieties $\mathcal{V}\left(A_{0} A_{m}\right)$ and $\mathcal{V}\left(A_{0} A_{m}, B_{0}\right)$ are the union of two intersected lines and a double point. The sequence implies $H_{\mathrm{c}}^{1}(U) \cong \mathbb{Q}(0)$ and $H_{\mathrm{c}}^{2}(U)=$ $Q(-1)^{\oplus 2}$. So, finishing our computation, we write

$$
\operatorname{gr}_{i}^{W} H_{\mathrm{c}}^{q}(U)= \begin{cases}Q(0), & q=1, \quad i=0, \\ 0, & q=1, \quad i=1, \\ 0, & q>1, \quad i=0,1\end{cases}
$$


In the opposite case, when $t \not \equiv 0 \bmod 2$, the matrix looks like

$$
\mathcal{M}_{\mathrm{GZZ}}=\left(\begin{array}{cccc}
B_{0} & A_{0} & \vdots & A_{m} \\
A_{0} & C_{1} & \vdots & A_{m-1} \\
\ldots & \ldots & \ddots & \vdots \\
A_{m} & A_{m-1} & \ldots & \ddots
\end{array}\right),
$$

and we had come from Step 3 or Step 6 . We deal with $U \subset \mathbb{P}^{2}\left(A_{0}: A_{m}: B_{0}\right)$ defined by

$$
\begin{aligned}
U & :=\mathcal{V}\left(A_{0} N_{1}\right) \backslash \mathcal{V}\left(A_{0} N_{1}, I_{1}\right) \\
& =\mathcal{V}\left(A_{0}\left(B_{0}+A_{0}-A_{m}\right)\right) \backslash \mathcal{V}\left(A_{0}\left(B_{0}+A_{0}-A_{m}\right), B_{0}\right)
\end{aligned}
$$

Changing the variables $A_{m}:=B_{0}+A_{0}-A_{m}$, we come to the situation above, and we again obtain the same result as (4.104).

We have constructed a sequence of schemes $U=U^{0}, U^{1}, \ldots, U^{n-4}=U_{3}$ (see (4.49)) such that $\operatorname{gr}_{i}^{W} H_{\mathrm{c}}^{q}\left(U^{s}\right) \cong \operatorname{gr}_{i}^{W} H_{\mathrm{c}}^{q-2}\left(U^{s-1}\right)$, for $0 \leq s \leq n-3$, $i=0,1, U^{s} \subset \mathbb{P}^{2 s+2}$, and $q=q(s) \geq 2 s+1$. By (4.50), we obtain

$$
\begin{aligned}
\operatorname{gr}_{i}^{W} H_{\operatorname{prim}}^{r-4}(V) & \cong \operatorname{gr}_{i}^{W} H_{\mathrm{c}}^{r-5}\left(U_{3}\right) \\
& \cong \operatorname{gr}_{i}^{W} H_{\mathrm{c}}^{r-5}\left(U^{n-4}\right) \cong \ldots \cong \operatorname{gr}_{i}^{W} H_{\mathrm{c}}^{q}\left(U^{0}\right), \quad i=0,1,
\end{aligned}
$$

where $q=r-5-2(n-4)=r-2 n+3 \geq 2 n-2-2 n+3=1$. Hence,

$$
\operatorname{gr}_{i}^{W} H_{\text {prim }}^{r-4}(V)= \begin{cases}Q(0), & r=2 n-2, \quad i=0 \\ 0, & r=2 n-2, \quad i=1 \\ 0, & r>2 n-2, \quad i=0,1\end{cases}
$$

Using the isomorphisms

$$
\operatorname{gr}_{i}^{W} H^{2 n-2}(X) \cong \operatorname{gr}_{i-4}^{W} H^{2 n-6}(V)(-2), \quad i=0, \ldots, 5
$$

(see (4.30)), we finally get

$$
\operatorname{gr}_{i}^{W} H_{\text {prim }}^{r}(X)= \begin{cases}Q(-2), & r=2 n-2, \quad i=4 \\ 0, & r=2 n-2, \quad i=0, \ldots, 3,5 \\ 0, & r>2 n-2, \quad i=0, \ldots, 5\end{cases}
$$


Recall that $\mathrm{ZZ}_{5}$ is a primitively log divergent graph and the smallest graph in the zigzag series (see, for example, [1, Section 1]) which is not isomorphic to a $\mathrm{WS}_{n}$ graph for some $n$.

Theorem 4.3. Let $X \subset \mathbb{P}^{9}$ be the hypersurface associated to $\mathrm{ZZ}_{5}$ (see Example 2.1 for the definition), then

$$
H_{\text {prim }}^{8}\left(Z Z_{5}\right) \cong \mathbb{Q}(-2) \text {. }
$$

Proof. We use the same stratification as for an arbitrary GZZ graph, but now we need to apply the more strong vanishing Theorems A and B to compute the whole cohomology of the middle degree rather than just the graded pieces.

In the case $\mathrm{ZZ}_{5}$, we have $n=5$ and $r=2 n-2=\operatorname{mid}=8$, and we start with the same localization sequence as in (4.14). Applying Theorem B $(N=$ $8, k=0, t=2)$ to $\mathbb{P}^{8} \backslash \mathcal{V}\left(I_{4}\right) \cong X \backslash \mathcal{V}\left(I_{5}, I_{4}\right)\left(I_{4}\right.$ independent of $A_{4}$ and $\left.A_{5}\right)$, we get $H^{i}\left(X \backslash \mathcal{V}\left(I_{5}, I_{4}\right)\right)=0$ for $i<10$. Thus, the sequence (4.14) implies

$$
H^{8}(X) \cong H^{8}\left(\mathcal{V}\left(I_{5}, I_{4}\right)\right) \cong H^{6}\left(\mathcal{V}\left(I_{4}, G_{4}\right)\right)(-1),
$$

where the variety on the right-hand side lives in $\mathbb{P}^{8}$ (no $B_{4}$ ) (compare with (4.22)). Since $I_{3}$ is independent of $A_{5}$ and $A_{4}$ (after $\left.C_{2}:=A_{2}\right)$, Theorem $\mathrm{B}$ $(N=6, k=0, t=2)$ implies $H^{i}\left(\mathbb{P}^{6} \backslash \mathcal{V}\left(I_{3}\right)\right)=H^{i}\left(\mathcal{V}\left(I_{4}, G_{4}\right) \backslash \hat{V}\right)=0$ for $i<$ 8 , where $\hat{V}:=\mathcal{V}\left(I_{4}, I_{3}, G_{4}\right) \subset \mathbb{P}^{8}$ (see (4.26)). The sequence (4.23) yields an isomorphism $H^{6}\left(\mathcal{V}\left(I_{4}, G_{4}\right)\right) \cong H^{6}(\hat{V})$. Combining with (4.112) and (4.28), we get

$$
H^{8}(X) \cong H^{4}(V)(-2),
$$

where $V:=\mathcal{V}\left(I_{4}, I_{3}, G_{4}^{\prime}\right) \subset \mathbb{P}^{7}\left(\right.$ no $\left.B_{4}, A_{4}\right)$. Here

$$
G_{4}^{\prime}:=\left.G_{4}\right|_{A_{3}=0}=A_{4}^{2} B_{3} I_{2}+A_{5}^{2} I_{3}^{1}-2 A_{4} A_{5}\left(B_{3} A_{0} A_{1}\right) .
$$

The same formula as (4.113) holds for the cohomology of the middle degree of arbitrary GZZ.

Now we get rid of $B_{3}$. We rewrite $G_{4}^{\prime}=B_{3} G_{3}-A_{2}^{2}\left(A_{5}^{2} B_{1}\right)$, where

$$
G_{3}:=A_{4}^{2} I_{2}+A_{5}^{2} I_{2}^{1}-2 A_{4} A_{5}\left(A_{0} A_{1}\right) .
$$

One has the localization sequence

$$
\rightarrow H^{1}(T)(-1) \rightarrow H_{\mathrm{c}}^{4}(V \backslash \hat{T}) \rightarrow H_{\text {prim }}^{4}(V) \rightarrow H_{\text {prim }}^{2}(T)(-1) \rightarrow
$$


for $T=V \cap \mathcal{V}\left(G_{3}\right)=\mathcal{V}\left(I_{3}, A_{2} I_{2}, G_{3}, A_{2} A_{5} B_{1}\right) \subset \mathbb{P}^{6}$ (no $\left.B_{4}, A_{4}, B_{3}\right)$. By Theorem $\mathrm{B}$, the cohomology to the left dies. Considering the localization sequence for $T \cap \mathcal{V}\left(A_{2}\right) \subset T$, one can see that

$$
H_{\text {prim }}^{2}(T) \cong H_{\mathrm{c}}^{2}\left(T \backslash T \cap \mathcal{V}\left(A_{2}\right)\right) .
$$

The scheme $T \backslash T \cap \mathcal{V}\left(A_{2}\right)$ is defined by

$$
\left\{\begin{array} { r } 
{ I _ { 3 } = A _ { 2 } I _ { 2 } = 0 , } \\
{ G _ { 3 } = A _ { 2 } A _ { 5 } B _ { 1 } = 0 , } \\
{ A _ { 2 } \neq 0 , }
\end{array} \Leftrightarrow \left\{\begin{array}{r}
I_{3}=I_{2}=0 \\
G_{3}=A_{5} B_{1}=0 \\
A_{2} \neq 0 .
\end{array}\right.\right.
$$

Define $R:=\mathcal{V}\left(I_{3}, I_{2}, G_{3}, A_{5} B_{1}\right)$ and $R_{1}=R \cap \mathcal{V}\left(A_{2}\right)$ in $\mathbb{P}^{6}$ (no $B_{4}, A_{3}, B_{3}$ ). We get an exact sequence

$$
\rightarrow H^{1}(R) \rightarrow H^{1}\left(R_{1}\right) \rightarrow H_{\mathrm{c}}^{2}\left(T \backslash T \cap \mathcal{V}\left(A_{2}\right)\right) \rightarrow H_{\text {prim }}^{2}(R) \rightarrow
$$

Applying Theorem 1.3 and using (4.115), one gets

$$
\begin{aligned}
R & =\mathcal{V}\left(I_{3}, I_{2}, G_{3}, A_{5} B_{1}\right)=\mathcal{V}\left(I_{3}, I_{2}, A_{5}^{2}\left(B_{1} C_{2}-A_{1}^{2}\right), A_{5} B_{1}\right) \\
(4.120) & =\mathcal{V}\left(C_{2} I_{2}-A_{1}^{2} B_{0}, I_{2}, A_{5} A_{1}, A_{5} B_{1}\right)=\mathcal{V}\left(A_{1} B_{0}, I_{2}, A_{5} A_{1}, A_{5} B_{1}\right) .
\end{aligned}
$$

The last polynomials are independent of $A_{2}$ and $A_{4}$, Theorem $\mathrm{A}(N=6, k=$ $4, t=2)$ implies $H_{\text {prim }}^{i}(R)=0$ for $i<4$. Moreover, the defining polynomials of $R_{1}$ do not depend on $A_{4}$, thus the cohomology $H^{1}\left(R_{1}\right)$ also vanishes. By (4.116), (4.117) and (4.119), we obtain

$$
H_{\text {prim }}^{4}(V) \cong H_{\mathrm{c}}^{4}(V \backslash \hat{T})
$$

(compare with (4.34)). The polynomials $I_{3}$ and $A_{2} I_{2}$ are independent of $A_{5}$, by Theorem $\mathrm{A}(N=6, k=2, t=1)$ for $\mathcal{V}\left(I_{3}, A_{2} I_{2}\right)$, the sequence $(4.37)$ implies

$$
H_{\mathrm{c}}^{4}(V \backslash \hat{T}) \cong H_{\mathrm{c}}^{4}\left(U_{1}\right) \cong H_{\text {prim }}^{3}\left(\mathcal{V}\left(I_{3}, A_{2} I_{2}, G_{3}\right)\right)
$$

After the change of variables $\left(C_{2}:=A_{2}\right)$, the defining polynomials of $\hat{S}:=$ $\mathcal{V}\left(I_{3}, I_{2}, G_{3}\right) \subset \mathbb{P}^{6}$ become independent of $A_{4}$. By Theorem A $(N=6, k=3$, 
$t=1)$, the sequence (4.39) yields

$$
H^{3}\left(\mathcal{V}\left(I_{3}, A_{2} I_{2}, G_{3}\right)\right) \cong H_{c}^{3}\left(U_{2}\right) .
$$

Solving the equations of $U_{2}^{\prime}$ (see (4.45)) on $A_{4}$, one gets $U_{2}^{\prime} \cong W \backslash Z$, where $W:=\mathcal{V}\left(A_{1} I_{2}+A_{5} A_{0} A_{1}-A_{1}^{2} B_{0}\right)=\mathcal{V}\left(A_{1} N_{2}\right)$ and $Z=W \cap \mathcal{V}\left(I_{2}\right)$. Collecting (4.113), (4.121), (4.122), (4.123) and (4.43), we obtain

$$
H_{\text {prim }}^{4}(V) \cong H_{\mathrm{c}}^{4}(V \backslash \hat{T}) \cong H_{\mathrm{c}}^{3}\left(U_{2}\right) \cong H_{\mathrm{c}}^{3}(W \backslash Z) .
$$

Consider an exact sequence

$$
\begin{aligned}
\rightarrow & H_{\mathrm{c}}^{2}\left(\mathcal{V}\left(A_{1}\right) \backslash \mathcal{V}\left(A_{1}, I_{2}\right)\right) \oplus H_{\mathrm{c}}^{2}\left(W_{1} \backslash Z_{1}\right) \rightarrow H_{\mathrm{c}}^{2}\left(\mathcal{V}\left(A_{1}\right) \cap W_{1} \backslash Z_{1}\right) \rightarrow \\
& H_{\mathrm{c}}^{3}(W \backslash Z) \rightarrow H_{\mathrm{c}}^{3}\left(\mathcal{V}\left(A_{1}\right) \backslash \mathcal{V}\left(A_{1}, I_{2}\right)\right) \oplus H_{\mathrm{c}}^{3}\left(W_{1} \backslash Z_{1}\right) \rightarrow,
\end{aligned}
$$

with $W_{1}=\mathcal{V}\left(N_{2}\right)$ and $Z_{1}=W_{1} \cap \mathcal{V}\left(I_{2}\right)$ in $\mathbb{P}^{4}$. Immediately, $H_{c}^{2}\left(W_{1} \backslash Z_{1}\right)=0$. Moreover, one has the localization sequence

$$
\rightarrow H_{\text {prim }}^{2}\left(W_{1}\right) \rightarrow H_{\text {prim }}^{2}\left(Z_{1}\right) \rightarrow H_{\mathrm{c}}^{3}\left(W_{1} \backslash Z_{1}\right) \rightarrow H^{3}\left(W_{1}\right) \rightarrow \text {. }
$$

The term on the left-hand side vanishes, and for the right-hand side we consider the localization sequence for $W_{1} \cap \mathcal{V}\left(A_{0}\right) \subset W_{1}$. It is easy to see that $W_{1} \cap \mathcal{V}\left(A_{0}\right)$ is a cone over double point while its complement is isomorphic to $\mathbb{A}^{3}$. Thus, the sequence implies $H^{3}\left(W_{1}\right)=0$. By $(4.126), H_{\mathrm{c}}^{3}\left(W_{1} \backslash Z_{1}\right) \cong$ $H_{\text {prim }}^{2}\left(Z_{1}\right)$. We can consider the localization sequence for $Z_{1} \cap \mathcal{V}\left(B_{0}\right) \subset Z_{1}$. It turns out that $Z_{1} \cap \mathcal{V}\left(B_{0}\right) \cong \mathbb{P}^{2}$ and $Z_{1} \backslash Z_{1} \cap \mathcal{V}\left(B_{0}\right) \cong \mathbb{A}^{2}$. Hence, $H_{\mathrm{c}}^{3}\left(W_{1} \backslash Z_{1}\right)$ $\cong H_{\text {prim }}^{2}\left(Z_{1}\right)=0$. We return to the sequence (4.125). Theorem B $(N=4$, $k=1, t=1)$ implies $H_{\mathrm{c}}^{i}\left(\mathcal{V}\left(A_{1}\right) \backslash \mathcal{V}\left(A_{1}, I_{2}\right)\right)=0$ for $i<4$. Thus, the sequence yields

$$
H_{\mathrm{c}}^{3}(W \backslash Z) \cong H_{\mathrm{c}}^{2}\left(\mathcal{V}\left(A_{1}\right) \cap W_{1} \backslash Z_{1}\right) .
$$

Forgetting $A_{1}$, we get $\mathcal{V}\left(A_{1}\right) \cap W_{1} \backslash Z_{1} \cong W_{2} \backslash Z_{2}$ with the latter living in $\mathbb{P}^{3}\left(A_{0}: A_{5}: B_{0}: B_{1}\right)$. Stratification further gives us no result, so some geometrical argument must be involved at this step.

The variety $W_{2} \subset \mathbb{P}^{3}\left(A_{0}: A_{5}: B_{0}: B_{1}\right)$ is a smooth quadric. Up to a change of variables $W_{2}$ is the image of Segre imbedding. More precisely, 
$W_{2}=\operatorname{Im}(\gamma)$ for

$$
\gamma: \mathbb{P}^{1} \times \mathbb{P}^{1} \hookrightarrow \mathbb{P}^{3}:(a: b),(c: d) \mapsto(a c: a c-b d: a d: b c)
$$

Now, $Z_{2} \subset W_{2} \subset \mathbb{P}^{3}$ is defined by $Z_{2}:=\mathcal{V}\left(A_{0} A_{5}, B_{0} B_{1}-A_{0}^{2}\right)$. So $Z_{2}$ is a union of three components $\ell_{1} \cup \ell_{2} \cup \ell_{3}$, where $\ell_{1}$ and $\ell_{2}$ coincide with the lines $\gamma\left(\{\infty\} \times \mathbb{P}^{1}\right)$ and $\gamma\left(\mathbb{P}^{1} \times\{\infty\}\right)$, respectively, and $\ell_{3}$ is a zero of a nontrivial section of $\mathcal{O}(1,1)$. If

$$
S \backslash\left(\ell_{1} \cup \ell_{2}\right) \cong \mathbb{P}^{1} \times \mathbb{P}^{1} \backslash\left(\mathbb{P}^{1} \times\{\infty\} \cup\{\infty\} \times \mathbb{P}^{1}\right)=\mathbb{A}^{2}
$$

has affine coordinates $b, d$, then $\ell_{3} \cap \mathbb{A}^{2}$ has defining ideal $\langle 1-b d\rangle$, so is isomorphic to $\mathbb{G}_{m}$. Thus, we get $W_{2} \backslash Z_{2} \cong \mathbb{A}^{2} \backslash \mathbb{G}_{m}$. Now it follows that

$$
H_{\mathrm{c}}^{2}\left(W_{2} \backslash Z_{2}\right) \cong H_{\mathrm{c}}^{2}\left(\mathbb{A}^{2} \backslash \mathbb{G}_{m}\right) \cong H_{\mathrm{c}}^{1}\left(\mathbb{G}_{m}\right) \cong \mathbb{Q}(0)
$$

By (4.113), (4.124) and (4.127), we finally get

$$
H_{\text {prim }}^{8}(X) \cong H_{\mathrm{c}}^{3}(W \backslash Z)(-2) \cong \mathbb{Q}(-2)
$$

Theorem 4.4. Let $X$ be the hypersurface associated to a GZZ and $q=p^{k}$ some prime power. Then

$$
\# X\left(\mathbb{F}_{q}\right) \equiv 1+q+2 q^{2} \bmod q^{3}
$$

Proof. We use the same stratification as in the proof of Theorem 4.2 but translate everything into another language. Now we need $\ell$-adic cohomology. The h.i. for some $\hat{Y}, H^{q}(\hat{Y}) \cong H^{q-2}(Y)(-1)$ will now mean "Eigenvalues $(\mathrm{EV})$ of $F^{k}$ on $H^{q}(\hat{Y})$ are that ones on $H^{q-2}(Y)$ multiplied by $q$," where $F$ is the geometric Frobenius element (see [7, p. 26]). This is because $\mathrm{EV}\left(F^{k} \mid H_{\mathrm{c}}^{2}\left(\mathbb{A}^{1}\right)\right)=\{q\}$. Eigenvalues are a priori living in $\overline{\mathbb{Z}}$ (see $\left.[8,3.3 .4]\right)$. Assume now that we have an exact sequence like

$$
\longrightarrow H_{\mathrm{c}}^{q-2}(U)(-1) \longrightarrow H^{q}(Z) \longrightarrow H^{q}(T) \longrightarrow H_{\mathrm{c}}^{q-1}(U)(-1) \longrightarrow
$$

with $T \subset Z \subset \mathbb{P}^{N}$ and $Z \backslash T$ a cone over $U$. This is the example of a sequence which we usually deal with in the proof above. Instead of applying the $\operatorname{gr}_{i}^{W}$ functors, we take the eigenvalues of the action $F^{k}$. One sees that an 
eigenvalue of $F^{k} \mid H^{q}(Z)$ is that of $F^{k} \mid H^{q}(T)$ or in $E V\left(F^{k} \mid H_{\mathrm{c}}^{q-2}(U)(-1)\right)=$ $q \cdot E V\left(F^{k} \mid H_{\mathrm{c}}^{q-2}(U)\right)$. For the sequence (4.133), we can write

$$
E V\left(F^{k} \mid H^{q}(Z)\right)=E V\left(F^{k} \mid H^{q}(T)\right) \bmod q \cdot \overline{\mathbb{Z}}
$$

We play the same game in each situation where we use $\mathrm{gr}_{i}^{W}$ functors in the proof of Theorem 4.2. We get the following result: all EV of $F^{k} \mid H_{\text {prim }}^{\operatorname{mid}}(X)$ are in $q^{3} \cdot \overline{\mathbb{Z}}$ but one is exactly $q^{2}$; all EV of $F^{k} \mid H_{\text {prim }}^{r}(X)$ are in $q^{3} \cdot \overline{\mathbb{Z}}$ if $r>$ mid. Finally, we use the Grothendick-Lefschetz trace formula and get

$$
\begin{aligned}
\# X\left(\mathbb{F}_{q}\right)= & \sum_{i=0}^{4 n-4}(-1)^{i} \operatorname{Tr}\left(F^{k} \mid H^{i}\left(X_{\Gamma}\right)\right)=1+q+\cdots+q^{2 n-2} \\
& +\sum_{i=2 n-2}^{4 n-4}(-1)^{i} \operatorname{Tr}\left(F^{k} \mid H_{\text {prim }}^{i}\left(X_{\Gamma}\right)\right) \equiv 1+q+2 q^{2} \bmod q^{3} .
\end{aligned}
$$

\section{De Rham class for $\operatorname{GZZ}(n, 2)$}

Fix some $n \geq 2$ and define $\Gamma=\Gamma_{n}:=\operatorname{GZZ}(n, 2)$. This graph has $2 n+6$ edges and $h_{1}(\Gamma)=n+3$. Let $X_{n} \subset \mathbb{P}^{2 n+5}$ be the graph hypersurface associated to $\Gamma_{n}$. By the results of the previous section, one has an inclusion

$$
\mathbb{Q}(-2) \hookrightarrow H_{\mathrm{prim}}^{2 n+4}\left(X_{n}\right) \cong H_{\mathrm{c}}^{2 n+5}\left(\mathbb{P}^{2 n+5} \backslash X\right)
$$

Hence, we get $\operatorname{dim} H_{\mathrm{DR}}^{2 n+5}\left(\mathbb{P}^{2 n+5} \backslash X_{n}\right) \neq 0$. We do not know that this cohomology group is one-dimensional in general. In this section we consider

$$
\eta=\eta_{n}=\frac{\Omega_{2 n+5}}{\Psi_{\Gamma_{n}}^{2}} \in \Gamma\left(\mathbb{P}^{2 n+5}, \omega\left(2 X_{n}\right)\right)
$$

(see $(2.9))$ and show that $\left[\eta_{n}\right] \neq 0$ in $H^{2 n+5}\left(\mathbb{P}^{2 n+5} \backslash X_{n}\right)$. We strongly follow $\left[2\right.$, Section 12], where the computations for $\mathrm{WS}_{n}$ were done.

Lemma 5.1. Let $U=\operatorname{Spec} R$ be a smooth, affine variety and $0 \neq f, g \in R$. Define $Z:=\mathcal{V}(f, g) \subset U$. We have a map of complexes

$$
\left(\Omega_{R[1 / f]}^{*} / \Omega_{R}^{*}\right) \oplus\left(\Omega_{R[1 / g]}^{*} / \Omega_{R}^{*}\right) \stackrel{\gamma}{\longrightarrow}\left(\Omega_{R[1 / f g]}^{*} / \Omega_{R}^{*}\right) .
$$

Then the de Rham cohomology with supports $H_{Z, \mathrm{DR}}^{*}(U)$ can by computed by the cone of $\gamma$ shifted by -2 . 
Remark 5.1. The direct computation shows that $C^{*}$ is quasi-isomorphic to the cone of

$$
\left(\Omega_{R[1 / f]}^{*} / \Omega_{R}^{*}\right) \stackrel{\Delta}{\longrightarrow}\left(\Omega_{R[1 / f g]}^{*} / \Omega_{R[1 / g]}^{*}\right) .
$$

For the application, we use $U:=\mathbb{P}^{2 n+5} \backslash X_{n}$. Recall that the matrix of $\Gamma_{n}$ looks like

$$
\mathcal{M}_{\Gamma_{n}}=\left(\begin{array}{cccccccc}
B_{0} & A_{0} & 0 & \vdots & 0 & 0 & 0 & A_{n+3} \\
A_{0} & B_{1} & A_{1} & \vdots & 0 & 0 & 0 & 0 \\
0 & A_{1} & B_{6} & \vdots & 0 & 0 & 0 & 0 \\
\cdots & \ldots & \ldots & \ddots & \ldots & \ldots & \ldots & \ldots \\
0 & 0 & 0 & \vdots & B_{n-1} & A_{n-1} & 0 & 0 \\
0 & 0 & 0 & \vdots & A_{n-1} & C_{n} & A_{n} & A_{n+2} \\
0 & 0 & 0 & \vdots & 0 & A_{n} & B_{n+1} & A_{n+1} \\
A_{n+3} & 0 & 0 & \vdots & 0 & A_{n+2} & A_{n+1} & B_{n+2}
\end{array}\right) .
$$

Define $a_{i}:=\frac{A_{i}}{A_{n+3}}, b_{i}:=\frac{B_{i}}{A_{n+3}}$ and $c_{n}:=\frac{C_{n}}{A_{n+3}}=a_{n+2}+a_{n-1}-a_{n}$. (We will see that the forms we work with have no poles along $A_{n+3}=0$.) Let

$$
i_{j}=\frac{I_{j}}{A_{n+3}^{j}}, \quad g_{n+2}=\frac{G_{n+2}}{A_{n+3}^{n+3}} .
$$

Set $f=i_{n+2}, g=i_{n+1}$. The equation $b_{n+2} i_{n+2}-g_{n+2}=0$ defines $X_{n}$ on $A_{n+3} \neq 0$, then $b_{n+2} i_{n+2}-g_{n+2}$ is invertible on $U=\mathbb{P}^{2 n+5} \backslash X$. Thus, $g_{n+2}$ is invertible on $\mathcal{V}(f)$. The element

$$
\begin{aligned}
\beta= & -d b_{0} \wedge \ldots \wedge d b_{n-1} \wedge d b_{n+1} \wedge \\
& \wedge d a_{0} \wedge \ldots \wedge d a_{n+2} \frac{1}{g_{n+2} i_{n+2}}\left(\frac{g_{n+2}}{b_{n+2} i_{n+2}-g_{n+2}}\right)
\end{aligned}
$$

is defined in $\Omega_{R[1 / f]}^{2 n+4} / \Omega_{R}^{2 n+4}$. It satisfies

$$
d \beta=\eta=\frac{d b_{0} \wedge \ldots \wedge d b_{n-1} \wedge d b_{n+1} \wedge d b_{n+2} \wedge d a_{0} \wedge \ldots \wedge d a_{n+2}}{\left(b_{n+2} i_{n+2}-g_{n+2}\right)^{2}}
$$


By Corollary $1.3, I_{n+1} G_{n+2} \equiv\left(L_{n+2}\right)^{2} \bmod I_{n+2}$, thus

$$
\begin{aligned}
i_{n+1} g_{n+2} \equiv & \left(a_{n+1} i_{n+1}-a_{n+2} a_{n} i_{n}\right. \\
& \left.+(-1)^{n-1} a_{n} a_{n-1} \ldots a_{1} a_{0}\right)^{2} \bmod i_{n+2} .
\end{aligned}
$$

We also use

$$
i_{k}=b_{k-1} i_{k-1}-a_{k-2}^{2} i_{k-2}
$$

for $k=n+2$ or $k<n+1$. We now compute in $\Omega_{R[1 / f g]}^{*} / \Omega_{R[1 / g]}^{*}$ and get

$$
\begin{aligned}
\beta= & \frac{d i_{n+2}}{i_{n+2}} \wedge \frac{d a_{n+2}}{g_{n+2} i_{n+1}} \wedge d b_{0} \wedge \ldots \wedge d b_{n-1} \wedge \\
& \wedge d a_{0} \wedge \ldots \wedge d a_{n+1} \cdot\left(1-\frac{b_{n+2} i_{n+2}}{b_{n+2} i_{n+2}-g_{n+2}}\right) \\
= & -d\left(\frac{1}{a_{n+1} i_{n+1}-a_{n+2} a_{n} i_{n}+(-1)^{n-1} a_{n} \ldots a_{0}} \cdot \frac{d i_{n+2}}{i_{n+2}} \wedge \nu\right)
\end{aligned}
$$

where

$$
\nu:=\frac{d a_{n+2}}{i_{n+1}} \wedge d b_{0} \wedge \ldots \wedge d b_{n-1} \wedge d a_{0} \wedge \ldots \wedge d a_{n} .
$$

Using the equality

$$
i_{n+1}=c_{n} i_{n}-a_{n-1}^{2} i_{n-1}=\left(a_{n+2}+a_{n-1}-a_{n}\right) i_{n}-a_{n-1}^{2} i_{n-1}
$$

and (5.10), we get

$$
\begin{aligned}
\nu= & \frac{d i_{n+1}}{i_{n+1}} \wedge \frac{d b_{n-1}}{i_{n}} \wedge d b_{n-2} \wedge \ldots \wedge d b_{0} \wedge d a_{0} \wedge \ldots \wedge d a_{n} . \\
& \frac{d i_{n+1}}{i_{n+1}} \wedge \frac{d i_{n}}{i_{n}} \wedge \ldots \wedge \frac{d i_{2}}{i_{2}} \wedge \frac{d b_{0}}{b_{0}} \wedge d a_{0} \wedge \ldots \wedge d a_{n} .
\end{aligned}
$$

By (5.11) one has $\beta=d \theta$ with

$$
\theta:=-\frac{1}{a_{n+1} i_{n+1}-a_{n+2} a_{n} i_{n}+(-1)^{n-1} a_{n} \ldots a_{0}} \cdot \frac{d i_{n+2}}{i_{n+2}} \wedge \nu .
$$

Both $\beta$ and $\theta$ have no poles along $A_{n+3}=0$. Thus the pair

$$
(\beta, \theta) \in H_{Z, \mathrm{DR}}^{2 n+5}(U)
$$

(see Remark 5.1) represents a class mapping to $\left[\eta_{n}\right] \in H_{\mathrm{DR}}^{2 n+5}\left(\mathbb{P}^{2 n+5} \backslash X_{n}\right)$, where $Z:=\mathcal{V}\left(I_{n+2}, I_{n+1}\right)$. 
Lemma 5.2. The natural map

$$
H_{Z}^{2 n+5}\left(\mathbb{P}^{2 n+5} \backslash X_{n}\right) \longrightarrow H^{2 n+5}\left(\mathbb{P}^{2 n+5} \backslash X_{n}\right)
$$

is injective.

Proof. The proof repeats that of Lemma 12.3. in [2] because the first two reduction steps for all GZZ yield the same result (see 4.113).

Theorem 5.1. Let $X_{n}$ be the graph hypersurface for $\Gamma_{n}=\operatorname{GZZ}(n, 2)$ and let $\left[\eta_{n}\right] \in H_{\mathrm{DR}}^{2 n+5}\left(\mathbb{P}^{2 n+5} \backslash X\right)$ be the de Rham class of (5.2). Then $\left[\eta_{n}\right] \neq 0$.

Proof. The proof is almost the same as that of Theorem 12.4 in [2]. We have lifted the class $\left[\eta_{n}\right]$ to a class $(\beta, \eta) \in H^{2 n+5}\left(\mathbb{P}^{2 n+5} \backslash X\right)$, see $(5.16)$. By Lemma 5.2 , it is enough to show that $(\beta, \eta) \neq 0$. We localize the generic point of $Z$ and compute further in the function field of $Z$. Consider the long denominator of $\beta$ in (5.11):

$$
D:=a_{n+1} i_{n+1}-a_{n+2} a_{n} i_{n}+(-1)^{n-1} a_{n} \ldots a_{0} .
$$

On $\mathcal{V}\left(i_{n+2}, i_{n+1}\right)$ we have

$$
\left\{\begin{array} { r l } 
{ b _ { n + 1 } i _ { n + 1 } - a _ { n } ^ { 2 } i _ { n } } & { = 0 , } \\
{ i _ { n + 1 } } & { = 0 , }
\end{array} \Rightarrow \left\{\begin{array}{c}
a_{n} i_{n}=0 \\
i_{n+1}=0
\end{array}\right.\right.
$$

thus both the left and the middle summand of $D$ vanish. Now it follows that as the class in the function field of $Z$, the class $(\beta, \eta)$ is represented by

$$
\pm d \log \left(i_{n}\right) \wedge \ldots \wedge d \log \left(i_{1}\right) \wedge d \log \left(a_{0}\right) \wedge \ldots \wedge d \log \left(a_{n}\right)
$$

This is a nonzero multiple of

$$
d \log \left(b_{n-1}\right) \wedge \ldots \wedge d \log \left(b_{0}\right) \wedge d \log \left(a_{0}\right) \wedge \ldots \wedge d \log \left(a_{n}\right)
$$

so is nonzero as a form. The Deligne theory of MHS yields that the vector space of logarithmic forms injects into de Rham cohomology of the open on which those forms are smooth (see [5, (3.1.5.2)]). Thus the form above is nonzero.

Corollary 5.1. Let $X$ be the graph hypersurface for $\Gamma=Z Z_{5}$. Then the class of $\eta$ defined in (5.2) spans $H_{\mathrm{DR}}^{9}\left(\mathbb{P}^{9} \backslash X\right)$. 


\section{Gluings}

Analyzing the different possibilities for the adjacency matrix, one can easily classify the primitively divergent graphs with small number of edges.

Theorem 6.1. Let $\Gamma$ be a primitively divergent graph with $E(\Gamma)=2 n$ and $n \leq 5$. Then for $\Gamma$ we have one of the following possibilities:

- $n=3$, then $\Gamma \cong \mathrm{WS}_{3}$.

- $n=4$, then $\Gamma \cong \mathrm{WS}_{4}$.

- $n=5$, then $\Gamma$ is isomorphic to the one of the following graphs $\mathrm{WS}_{5}$, $\mathrm{ZZ}_{5}, \mathrm{XX}_{5}$ or $\mathrm{ST}_{5}$.

The last two graphs look like
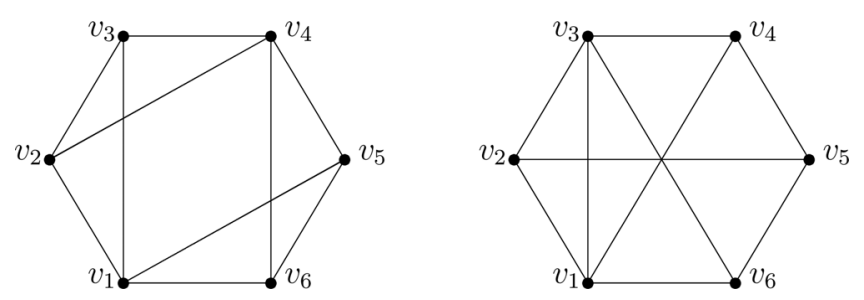

We cannot say anything important about the graph hypersurface of the (strange) graph $\mathrm{ST}_{5}$ on the cohomological level, but the graph $\mathrm{XX}_{5}$ motivates us to the following interesting construction.

Definition 6.1. Let $\Gamma$ and $\Gamma^{\prime}$ be two graphs, choose two edges $(u, v) \in E(\Gamma)$ and $\left(u^{\prime}, v^{\prime}\right) \in E\left(\Gamma^{\prime}\right)$. We define the graph $\Gamma \times \Gamma^{\prime}$ as follows. We drop the edges $(u, v)$ and $\left(u^{\prime}, v^{\prime}\right)$, and identify vertices $u$ with $u^{\prime}$ and $v$ with $v^{\prime}$. We also say that $\Gamma \times \Gamma^{\prime}$ is the gluing of $\Gamma$ and $\Gamma^{\prime}$ along edges $(u, v)$ and $\left(u^{\prime}, v^{\prime}\right)$.

Example 6.1. The graph $\mathrm{XX}$ appeared in Theorem 6.1 is isomorphic to $\mathrm{WS}_{3} \times \mathrm{WS}_{3}$.

It is not easy to verify whether the graph is primitively log divergent or not. Nevertheless, we can construct new primitively log divergent graphs from the existing ones by the gluing operation.

Theorem 6.2. The gluing $\Gamma \times \Gamma^{\prime}$ of two primitively log divergent graphs $\Gamma$ and $\Gamma^{\prime}$ (along edges $(u, v)$ and $\left.\left(u^{\prime}, v^{\prime}\right)\right)$ is again primitively log divergent. 
Proof. Suppose that $\Gamma$ and $\Gamma^{\prime}$ have $2 n$ and $2 m$ edges, respectively, then $h_{1}(\Gamma)$ and $h_{1}\left(\Gamma^{\prime}\right)=m$. We can choose a basis $\left\{\gamma_{1}, \ldots, \gamma_{n}\right\}$ of $H_{1}(\Gamma, \mathbb{Z})$ such that the edge $(u, v)$ only appears in $\gamma_{n}$. Indeed, we take any basis $\left\{\gamma_{1}, \ldots, \gamma_{n-1}\right\}$ of $H_{1}(\Gamma \backslash\{(u, v)\}, \mathbb{Z})$ and define $\gamma_{n}$ to be any loop containing $(u, v)$, then $\left\{\gamma_{1}, \ldots, \gamma_{n}\right\}$ form a basis of $H_{1}(\Gamma, \mathbb{Z})$. Similarly, we choose a basis $\delta_{1}, \ldots, \delta_{m}$ such that the only appearance of $\left(u^{\prime}, v^{\prime}\right)$ is in $\delta_{m}$. It follows that the loops $\left\{\gamma_{1}, \ldots, \gamma_{n-1}, \delta_{1}, \ldots, \delta_{m-1}, \gamma_{n} \times \delta_{m}\right\}$ form a basis of $H_{1}\left(\Gamma \times \Gamma^{\prime}, \mathbb{Z}\right)$. Thus, $\left|E\left(\Gamma \times \Gamma^{\prime}\right)\right|=2 n+2 m-2=2 h_{1}\left(\Gamma \times \Gamma^{\prime}\right)$ and $\Gamma \times \Gamma^{\prime}$ is logarithmically divergent.

To prove that $\Gamma \times \Gamma^{\prime}$ is primitively log divergent, we consider a proper subgraph $\Gamma_{0} \subset \Gamma \times \Gamma^{\prime}$ and define $\Gamma_{1}$ (respectively $\Gamma_{2}$ ) to be the graph $\Gamma_{0} \cap$ $\Gamma \cup\{(u, v)\}$ (respectively $\Gamma_{0} \cap \Gamma^{\prime} \cup\left\{\left(u^{\prime}, v^{\prime}\right)\right\}$ ). Because the graphs $\Gamma$ and $\Gamma^{\prime}$ are primitively log divergent, for the subgraphs $\Gamma_{1} \subset \Gamma$ and $\Gamma_{2} \subset \Gamma^{\prime}$ the inequalities

$$
\left|E\left(\Gamma_{1}\right)\right| \leq 2 h_{1}\left(\Gamma_{1}\right) \quad \text { and } \quad\left|E\left(\Gamma_{2}\right)\right| \leq 2 h_{1}\left(\Gamma_{2}\right)
$$

hold, and the inequalities become strict if subgraphs are proper. Since $\Gamma_{0}$ is the proper subgraph, at least one of the subgraphs $\Gamma_{1}, \Gamma_{2}$ is proper. Thus, we get

$$
\left|E\left(\Gamma_{1}\right)\right|+\left|E\left(\Gamma_{2}\right)\right|<2\left(h_{1}\left(\Gamma_{1}\right)+h_{1}\left(\Gamma_{2}\right)\right)
$$

The number of edges of $\Gamma_{0}$ equals $\left|E\left(\Gamma_{0}\right)\right|=\left|E\left(\Gamma_{1}\right)\right|+\left|E\left(\Gamma_{2}\right)\right|-2$ and one has an inequality $h_{1}\left(\Gamma_{1}\right)+h_{1}\left(\Gamma_{2}\right)-1 \leq h_{1}\left(\Gamma_{0}\right)$, which becomes an equality if the operation of adding $(u, v)$ to $\Gamma_{0} \cap \Gamma$ (or that of $\left(u^{\prime}, v^{\prime}\right)$ to $\Gamma_{0} \cap \Gamma^{\prime}$ ) increases the Betti number. The inequality (6.2) implies $\left|E\left(\Gamma_{0}\right)\right|<2 h_{1}\left(\Gamma_{0}\right)$. Thus, every proper subgraph of $\Gamma \times \Gamma^{\prime}$ is convergent.

Corollary 6.1. Every gluing $\Gamma$ of finitely many GZZ graphs (along any pair of edges) is primitively log divergent.

Our goal here is to analyze the middle dimensional (Betti) cohomology of hypersurfaces associated to graphs $\mathrm{WS}_{n} \times \mathrm{WS}_{3}$ for $n \geq 3$. The gluing for $\mathrm{WS}_{n} \times \mathrm{WS}_{3}$ goes along some two boundary edges (not spokes).

Theorem 6.3. Let $X$ be the graph hypersurface for the graph $\mathrm{WS}_{n} \times \mathrm{WS}_{3}$, $n \geq 3$. For the cohomology of the middle degree $H^{\mathrm{mid}}(X)$, one has

$$
\operatorname{gr}_{6}^{W}\left(H_{\mathrm{prim}}^{\operatorname{mid}}(X)\right)=\mathbb{Q}(-3) \quad \text { and } \quad g r_{8}^{W}\left(H_{\mathrm{prim}}^{\mathrm{mid}}(X)\right)=\mathbb{Q}(-4)^{\oplus d},
$$

where $d=0,1$ or 2, and all other $\operatorname{gr}_{i}^{W}=0$. If $n=3$, then $d=0$. 
Proof. Fix $n \geq 3$ and consider the graph $\mathrm{WS}_{n}$. We orient the spokes $\left(v_{0}, v_{i}\right)$ as exiting the center $v_{0}$ and label them with $e_{1}$ through $e_{n}$. The boundary edges $\left(v_{i}, v_{i+1}\right)$ (modulo $n$ ) are denoted by $e_{n+i}$ and are oriented exiting $v_{i}$. Now we rename the last edge $e_{2 n}=: e$, play the same game with the graph $\mathrm{WS}_{3}$, shifting the numeration of edges by $2 n-1$, and glue $\mathrm{WS}_{n}$ with $\mathrm{WS}_{3}$ along $e$ and $e_{2 n+5}$. Denote the resulting graph by $\Gamma$. To show the way of constructing the tables and the matrices associated to this gluing, we restrict to the case $\mathrm{WS}_{4} \times \mathrm{WS}_{3}$.
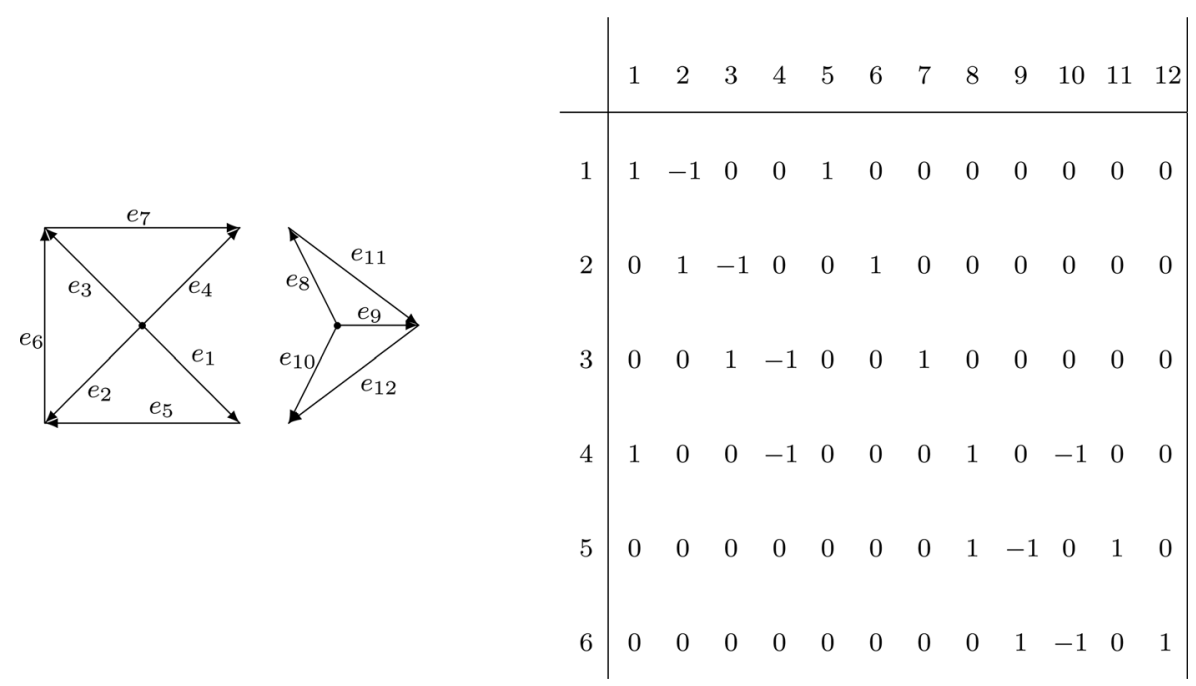

The matrix $\mathcal{M}_{\Gamma}$ has two "blocks" coming from the matrices of $\mathrm{WS}_{n}$ and $\mathrm{WS}_{3}$ intersected by one element which becomes dependent.

$$
\mathcal{M}_{\Gamma}(A, B)=\left(\begin{array}{ccccccccc}
B_{0} & A_{0} & 0 & \ldots & 0 & 0 & A_{n+1} & 0 & 0 \\
A_{0} & B_{1} & A_{1} & \ldots & 0 & 0 & 0 & 0 & 0 \\
0 & A_{1} & B_{2} & \ldots & 0 & 0 & 0 & 0 & 0 \\
\ldots & \ldots & \ldots & \ldots & \ldots & \ldots \ldots & \ldots \ldots \ldots \ldots \ldots \\
0 & 0 & 0 & \ldots & B_{n-3} & A_{n-3} & 0 & 0 & 0 \\
0 & 0 & 0 & \ldots & A_{n-3} & B_{n-2} & A_{n-2} & 0 & 0 \\
A_{n+1} & 0 & 0 & \ldots & 0 & A_{n-2} & C_{n-1} & A_{n-1} & A_{n+2} \\
0 & 0 & 0 & \ldots & 0 & 0 & A_{n-1} & B_{n} & A_{n} \\
0 & 0 & 0 & \ldots & 0 & 0 & A_{n+2} & A_{n} & B_{n+1}
\end{array}\right)
$$

where $C_{n-1}:=A_{n+1}+A_{n-2}+A_{n-1}+A_{n+2}$. We work with the graph hypersurface $X:=\operatorname{det} \mathcal{M}_{\Gamma} \subset \mathbb{P}^{2 n+3}(A, B)$, and the cohomology to compute is $H^{\text {mid }}(X)=H^{2 n+2}(X)$. We write $I_{n+2}=B_{n+1} I_{n+1}-G_{n+1}$. One has the 
localization sequence

$$
\begin{aligned}
& \longrightarrow H_{\mathrm{c}}^{2 n+2}(U) \longrightarrow H^{2 n+2}(X) \longrightarrow \\
& H^{2 n+2}\left(\mathcal{V}\left(I_{n+2}, I_{n+1}\right)\right) \longrightarrow H_{\mathrm{c}}^{2 n+3}(U) \longrightarrow,
\end{aligned}
$$

where $U:=X \backslash \mathcal{V}\left(I_{n+2}, I_{n+1}\right) \subset \mathbb{P}^{2 n+3}(A, B)$. For dimensional reasons, we have the vanishing of the term on the right-hand side. For the rightmost term, one easily gets

$$
\begin{aligned}
H_{\mathrm{c}}^{2 n+3}(U) \cong & H_{\mathrm{c}}^{2 n+3}\left(\mathbb{P}^{2 n+2} \backslash \mathcal{V}\left(I_{n+1}\right)\right) \\
& \cong H_{\mathrm{c}}^{2 n+1}\left(\mathbb{P}^{2 n+1} \backslash \mathcal{V}\left(I_{n+1}\right)\right)(-1) \cong H^{2 n}\left(\mathcal{V}\left(I_{n+1}^{\prime}\right)\right)(-1)
\end{aligned}
$$

Prime indicates that we made the change of coordinates $C_{n-1}:=A_{n+2}$. We consider an exact sequence

$$
\longrightarrow H_{\mathrm{c}}^{2 n}\left(T_{0}\right) \longrightarrow H_{\mathrm{prim}}^{2 n}\left(\mathcal{V}\left(I_{n+1}^{\prime}\right)\right) \longrightarrow H_{\mathrm{prim}}^{2 n}(\hat{T}) \longrightarrow
$$

where $\hat{T}:=\mathcal{V}\left(I_{n+1}^{\prime}, I_{n}^{\prime}\right)$ and $T_{0}:=\mathcal{V}\left(I_{n+1}^{\prime}\right) \backslash \hat{T}$ in $\mathbb{P}^{2 n+1}$ (no $\left.B_{n+1}, A_{n}\right)$. Since $I_{n+1}^{\prime}=B_{n} I_{n}^{\prime}-A_{n-1}^{2} I_{n-1}$, one gets $T_{0} \cong \mathbb{P}^{2 n} \backslash \mathcal{V}\left(I_{n}^{\prime}\right)$ and Theorem $\mathrm{B}(N=2 n$, $k=0, t=1)$ implies $H_{\mathrm{c}}^{2 n}\left(T_{0}\right)=0$. For $\hat{T}$, one gets $H_{\text {prim }}^{2 n}(\hat{T}) \cong H_{\text {prim }}^{2 n-2}(T)(-1)$ with $T:=\mathcal{V}\left(I_{n}^{\prime}, A_{n-1} I_{n-1}\right) \subset \mathbb{P}^{2 n}\left(\right.$ no $\left.B_{n+1}, A_{n}, B_{n}\right)$.

Consider $T_{1}:=T \cap \mathcal{V}\left(I_{n-1}\right), T_{00}=T \backslash T_{1}$, and the localization sequence

$$
\longrightarrow H_{c}^{2 n-2}\left(T_{00}\right) \longrightarrow H_{\mathrm{prim}}^{2 n-2}(T) \longrightarrow H_{\mathrm{prim}}^{2 n-2}\left(T_{1}\right) \longrightarrow .
$$

The polynomials $I_{n}^{\prime}$ and $I_{n-1}$ are independent of $A_{n-1}$. Thus, Theorem A $(N=2 n, k=2, t=1)$ implies $H_{\text {prim }}^{2 n-2}\left(T_{1}\right)=0$. Solving the equation $I_{n}^{\prime}=$ $C_{n-1} I_{n-1}-G_{n-1}$ on $C_{n-1}$, one gets $T_{00} \cong \mathbb{P}^{2 n-1} \backslash \mathcal{V}\left(I_{n-1}\right)$. By Theorem B $(N=2 n-1, k=0, t=2)$, one obtains $H_{\mathrm{c}}^{2 n-2}\left(T_{00}\right)=0$.

The exact sequence (6.8) implies $H_{\text {prim }}^{2 n+2}(T)=0$. The sequence (6.7) and isomorphisms in $(6.6)$ yield $H_{\mathrm{c}}^{2 n+3}(U) \cong H_{\text {prim }}^{2 n}\left(\mathcal{V}\left(I_{n+1}^{\prime}\right)\right)(-1)=0$. We return to the sequence $(6.5)$ and get an isomorphism

$$
H^{2 n+2}(X) \cong H^{2 n+2}\left(\mathcal{V}\left(I_{n+2}, I_{n+1}\right)\right) \cong H^{2 n}\left(\mathcal{V}\left(I_{n+1}, G_{n+1}\right)\right)(-1)
$$

We consider $\hat{V}:=\mathcal{V}\left(I_{n+1}, G_{n+1}, I_{n}\right) \subset \mathcal{V}\left(I_{n+1}, G_{n+1}\right) \subset \mathbb{P}^{2 n+2}$ we write an exact sequence

$$
\longrightarrow H_{\mathrm{c}}^{2 n}\left(U_{1}\right) \longrightarrow H^{2 n}\left(\mathcal{V}\left(I_{n+1}, G_{n+1}\right)\right) \longrightarrow H^{2 n}(\hat{V}) \longrightarrow H_{\mathrm{c}}^{2 n+1}\left(U_{1}\right) \longrightarrow,
$$


where $U_{1}:=\mathcal{V}\left(I_{n+1}, G_{n+1}\right) \backslash \hat{V}$. Similar as in (4.26) and (4.27), we get $H_{\mathrm{c}}^{q}\left(U_{1}\right)$ $\cong H_{\mathrm{c}}^{q-2}\left(\mathbb{P}^{2 n-1} \backslash \mathcal{V}\left(I_{n}^{\prime}\right)\right)(-1) \cong H_{\text {prim }}^{q-3}\left(\mathcal{V}\left(I_{n}^{\prime}\right)\right)(-1)$ for $q=2 n, 2 n+1$. Note that $\mathcal{V}\left(I_{n}^{\prime}\right)$ is exactly the graph hypersurface for $\mathrm{WS}_{n}$. Thus the sequence (6.10) simplifies to

$$
0 \longrightarrow H^{2 n}\left(\mathcal{V}\left(I_{n+1}, G_{n+1}\right)\right) \longrightarrow H^{2 n}(\hat{V}) \longrightarrow \mathbb{Q}(-3) \longrightarrow .
$$

As in (4.28), one has

$$
H^{2 n}(\hat{V}) \cong H^{2 n-2}(V)(-1),
$$

where $V:=\mathcal{V}\left(I_{n}, I_{n+1}, A_{n+2} B_{n} I_{n-1}\right) \subset \mathbb{P}^{2 n+1}$ (no $\left.B_{n+1}, A_{n}\right)$.

Now we attack $V$. Rewriting $V=\mathcal{V}\left(I_{n}, A_{n+2} B_{n} I_{n-1}, A_{n-1} I_{n-1}\right)$, we define $V_{1}:=\mathcal{V}\left(I_{n}, B_{n}, A_{n-1} I_{n-1}\right)$ and $V_{2}:=\mathcal{V}\left(I_{n}, A_{n+2} I_{n-1}, A_{n-1} I_{n-1}\right)$ in $\mathbb{P}^{2 n+1}\left(\right.$ no $\left.B_{n+1}, A_{n}\right)$. Consider an exact sequence

$$
\begin{gathered}
\longrightarrow H^{2 n-3}\left(V_{1}\right) \oplus H^{2 n-3}\left(V_{2}\right) \longrightarrow H^{2 n-3}\left(V_{3}\right) \longrightarrow \\
H_{\text {prim }}^{2 n-2}(V) \longrightarrow H_{\text {prim }}^{2 n-2}\left(V_{1}\right) \oplus H_{\text {prim }}^{2 n-2}\left(V_{2}\right) \longrightarrow,
\end{gathered}
$$

with $V_{3}:=V_{1} \cap V_{2}=\mathcal{V}\left(I_{n}, B_{n}, A_{n+2} I_{n-1}, A_{n-1} I_{n-1}\right)$. The defining polynomials of $V_{2}$ are independent of $B_{n}$. Theorem A $(N=2 n+1, k=3, t=1)$ implies $H_{\text {prim }}^{i}\left(V_{2}\right)=0$ for $i \leq 2 n-2$. Considering $V_{11}:=\mathcal{V}\left(I_{n}, B_{n}, I_{n-1}\right) \subset$ $V_{1}$ and the associated localization sequence, one can also prove $H_{\text {prim }}^{i}\left(V_{2}\right)=0$ for $i \leq 2 n-2$. Thus, (6.13) implies

$$
H_{\text {prim }}^{2 n-2}(V) \cong H^{2 n-3}\left(V_{3}\right) .
$$

Define $V_{31}:=V_{3} \cap \mathcal{V}\left(I_{n-1}\right)=\mathcal{V}\left(I_{n}, B_{n}, I_{n-1}\right) \subset V_{3} \subset \mathbb{P}^{2 n+1}$ (no $B_{n+1}, A_{n}$ ). By Theorem A $(N=2 n+1, k=3, t=0), H_{\text {prim }}^{i}\left(V_{31}\right)=0$ for $i \leq 2 n-3$. Thus, the localization implies

$$
H^{2 n-3}\left(V_{3}\right) \cong H_{\mathrm{c}}^{2 n-3}\left(V_{3} \backslash V_{31}\right) .
$$

The subscheme $V_{3} \backslash V_{31}$ is defined by the system

$$
\left\{\begin{aligned}
A_{n-1} I_{n-1}=I_{n} & =0, \\
B_{n}=A_{n+2} I_{n-1} & =0, \\
I_{n-1} & \neq 0,
\end{aligned} \Leftrightarrow \quad \Leftrightarrow \quad \begin{array}{r}
A_{n-1}=I_{n}=0, \\
B_{n}=A_{n+2}=0, \\
I_{n-1} \neq 0 .
\end{array}\right.
$$

Define $Y:=\mathcal{V}\left(I_{n}\right), S:=\mathcal{V}\left(I_{n}, I_{n-1}\right)$ in $\mathbb{P}^{2 n-2}\left(\right.$ no $\left.\mathrm{DV}_{5}\right)$, where $\mathrm{DV}_{5}$ denotes the set of the dropped variables $\left\{B_{n+1}, A_{n}, B_{n}, A_{n+2}, A_{n-1}\right\}$. This gives us 
an exact sequence

$$
0 \longrightarrow H_{\mathrm{prim}}^{2 n-4}(S) \longrightarrow H^{2 n-3}\left(V_{3} \backslash V_{31}\right) \longrightarrow H^{2 n-3}(Y) \longrightarrow
$$

After rewriting $S=\mathcal{V}\left(I_{n-1}, G_{n-1}\right)$, we notice that $S$ is exactly the variety which appears in the first reduction step of the case of $\mathrm{WS}_{n}$, and we know that

$$
H^{2 n-4}(S) \cong \mathbb{Q}(-1)
$$

The computation of $H^{2 n-3}(Y)$ is less easy. The polynomial $I_{n}$ is similar to the polynomial associated to $\mathrm{WS}_{n}$ with the only difference that $C_{n-1}$ is not independent and is equal $A_{n+1}+A_{n-2}$. We start from the upper left corner of the matrix and write $I_{n}=B_{0} I_{n-1}^{1}-\widetilde{G}_{n-1}$. Consider $\hat{Y}_{1}:=\mathcal{V}\left(I_{n}, I_{n-1}^{1}\right) \subset$ $Y \subset \mathbb{P}^{2 n-2}\left(\right.$ no $\left.\mathrm{DV}_{5}\right)$. One has

$$
\rightarrow H_{\mathrm{c}}^{2 n-3}\left(Y \backslash \hat{Y}_{1}\right) \rightarrow H^{2 n-3}(Y) \rightarrow H^{2 n-3}\left(\hat{Y}_{1}\right) \rightarrow H_{\mathrm{c}}^{2 n-2}\left(Y \backslash \hat{Y}_{1}\right) \rightarrow
$$

For $Y \backslash \hat{Y}_{1}$ one gets

$$
H_{\mathrm{c}}^{q}\left(Y \backslash \hat{Y}_{1}\right) \cong H_{\mathrm{c}}^{q-2}\left(\mathbb{P}^{2 n-4} \backslash \mathcal{V}\left(I_{n-1}^{1}\right)(-1) \cong H_{\mathrm{prim}}^{q-3}\left(\mathcal{V}\left(I_{n-1}^{\prime 1}\right)\right)(-1)\right.
$$

for $q=2 n-3,2 n-2\left(C_{n-1}:=A_{n+1}\right)$. We see that $I_{n-1}^{1}$ is a determinant of a three-diagonal matrix studied in [2, Lemma 11.12], and its cohomology of middle degree is trivial. So, $H_{\text {prim }}^{q}\left(\mathcal{V}\left(I_{n-1}^{\prime 1}\right)\right)=0$ and the sequence (6.19) and h.i. yield

$$
H^{2 n-3}(Y) \cong H^{2 n-5}\left(Y_{1}\right)(-1)
$$

where $Y_{1}:=\mathcal{V}\left(I_{n-1}, G_{n-1}\right) \subset \mathbb{P}^{2 n-3}\left(\right.$ no $\left.\mathrm{DV}_{5}, B_{0}\right)$. Consider an exact sequence

$$
\rightarrow H_{\mathrm{c}}^{2 n-5}\left(Y_{1} \backslash \hat{Y}_{2}\right) \rightarrow H^{2 n-5}\left(Y_{1}\right) \rightarrow H^{2 n-5}\left(\hat{Y}_{2}\right) \rightarrow H_{\mathrm{c}}^{2 n-4}\left(Y_{1} \backslash \hat{Y}_{2}\right) \rightarrow
$$

where $\hat{Y}_{2}:=\mathcal{V}\left(I_{n-1}^{1}, \widetilde{G}_{n-1}, I_{n-2}^{2}\right)$. Similar to (4.26) (while interchanging rows and columns), projecting further, and changing the coordinates $\left(C_{n-1}:=\right.$ $\left.A_{n+1}\right)$, one gets $Y_{1} \backslash \hat{Y}_{2} \cong \mathcal{V}\left(I_{n-1}^{1}\right) \backslash \mathcal{V}\left(I_{n-1}^{1}, I_{n-2}^{2}\right) \cong \mathbb{P}^{2 n-5} \backslash \mathcal{V}\left(I_{n-2}^{\prime 2}\right)$. By the 
same argument as in (6.20), we obtain

$$
H_{\mathrm{c}}^{q}\left(Y_{1} \backslash \hat{Y}_{2}\right) \cong H_{\mathrm{c}}^{q-2}\left(\mathbb{P}^{2 n-6} \backslash \mathcal{V}\left(I_{n-2}^{\prime 2}\right)\right)(-1) \cong H_{\mathrm{prim}}^{q-3}\left(\mathcal{V}\left(I_{n-2}^{\prime 2}\right)\right)=0,
$$

for $q \leq 2 n-4$. After projecting further and using Theorem 1.3, we derive the following isomorphism from the sequence (6.22):

$$
H^{2 n-5}\left(Y_{1}\right) \cong H^{2 n-7}\left(Y_{2}\right)(-1),
$$

where $Y_{2}:=\mathcal{V}\left(I_{n-1}^{1}, A_{n+1} I_{n-2}^{1}, I_{n-2}^{2}\right) \subset \mathbb{P}^{2 n-4}\left(\right.$ no $\left.\mathrm{DV}_{5}, B_{0}, A_{0}\right)$. Now we see that if $n=3$, then we get the vanishing

$$
H^{2 n-7}\left(Y_{2}\right)=0 \quad \text { when } n=3 \text {. }
$$

From now on, we assume that $n \geq 4$. Define $Y_{21}:=\mathcal{V}\left(I_{n-1}^{1}, I_{n-2}^{1}, I_{n-2}^{2}\right), Y_{22}:=$ $\mathcal{V}\left(I_{n-1}^{1}, A_{n+1}, I_{n-2}^{2}\right)=\mathcal{V}\left(A_{n+1}, I_{n-2}^{2}, A_{1} I_{n-3}^{3}\right)$ and $Y_{3}:=Y_{21} \cap Y_{22}$. One has an exact sequence

$$
\begin{aligned}
\longrightarrow & H_{\mathrm{prim}}^{2 n-8}\left(Y_{21}\right) \oplus H_{\mathrm{prim}}^{2 n-8}\left(Y_{22}\right) \longrightarrow H_{\mathrm{prim}}^{2 n-8}\left(Y_{3}\right) \longrightarrow \\
& H^{2 n-7}\left(Y_{2}\right) \longrightarrow H^{2 n-7}\left(Y_{21}\right) \oplus H^{2 n-7}\left(Y_{22}\right) \longrightarrow
\end{aligned}
$$

By Theorem A $(N=2 n-4, k=3, t=1)$, we obtain $H^{q}\left(Y_{22}\right)=0$ for $q=$ $2 n-8,2 n-7$. The variety $Y_{21}$ (after $C_{n-1}:=A_{n+1}$ ) is exactly the variety that appeared in the proof of the $\mathrm{WS}_{n}$ case (was called $Z_{n-1}$, see $[2$, Theorem 11.9]). Thus $H^{2 n-7}\left(Y_{21}\right) \cong \mathbb{Q}(0)$ and the sequence (6.26) simplifies to

$$
0 \longrightarrow H_{\text {prim }}^{2 n-8}\left(Y_{3}\right) \longrightarrow H^{2 n-7}\left(Y_{2}\right) \longrightarrow \mathbb{Q}(0) \longrightarrow,
$$

where $Y_{3}:=\mathcal{V}\left(A_{n+1}, I_{n-1}^{1}, I_{n-2}^{1}, I_{n-2}^{2}\right)$. We change the notation and consider $Z:=\mathcal{V}\left(I_{n-1}^{1}, I_{n-2}^{1}, I_{n-2}^{2}\right) \subset \mathbb{P}^{2 n-5}\left(\right.$ no $\left.\mathrm{DV}_{8}\right)$, where $\mathrm{DV}_{8}:=\mathrm{DV}_{5} \cup\left\{B_{0}, A_{0}\right.$, $\left.A_{n+1}\right\}$. To abuse the notation, we write $I_{j}^{i}$ for $I_{j}^{i}$ after setting $A_{n+1}=0$ (so, $\left.C_{n-1}=A_{n-2}\right)$. We are interested in $H_{\text {prim }}^{2 n-8}(Z)$, the computation now goes in the same direction as in $\left[2\right.$, Theorem 11.9]. Consider $Z_{1}:=\mathcal{V}\left(I_{n-1}^{1}, I_{n-2}^{1}\right)$ and $Z_{2}:=\mathcal{V}\left(I_{n-1}^{1}, I_{n-2}^{2}\right)$ in $\mathbb{P}^{2 n-5}\left(\right.$ no $\left.\mathrm{DV}_{8}\right)$, then $Z=Z_{1} \cap Z_{2}$. We write an exact sequence

$$
\begin{aligned}
\longrightarrow & H_{\mathrm{prim}}^{2 n-8}\left(Z_{1}\right) \oplus H_{\mathrm{prim}}^{2 n-8}\left(Z_{2}\right) \longrightarrow H_{\mathrm{prim}}^{2 n-8}(Z) \longrightarrow \\
& H^{2 n-7}(\bar{Z}) \longrightarrow H^{2 n-7}\left(Z_{1}\right) \oplus H^{2 n-7}\left(Z_{2}\right) \longrightarrow
\end{aligned}
$$

where $\bar{Z}:=Z_{1} \cup Z_{2}$. Again by [2, Lemma 11.12], $H_{\text {prim }}^{q}\left(Z_{1}\right)=0$ for $q=2 n-$ $8,2 n-7$. Using induction, we can similarly prove $H_{\text {prim }}^{q}\left(Z_{2}\right)=0$. Thus, the 
sequence above gives us

$$
H_{\text {prim }}^{2 n-8}(Z) \cong H^{2 n-7}(\bar{Z})
$$

By Corollary 1.2, we get $\bar{Z}:=\mathcal{V}\left(I_{n-1}^{1}, I_{n-2}^{1} I_{n-2}^{2}\right)=\mathcal{V}\left(I_{n-1}^{1}, S_{n-2}\right)$ with $S_{n-2}:=$ $A_{1} A_{2} \ldots A_{n-2}$. Define $Z_{3}:=\mathcal{V}\left(I_{n-1}^{1}, A_{n-2}\right), Z_{4}:=\mathcal{V}\left(I_{n-1}^{1}, S_{n-3}\right)$, and $Z_{5}:=$ $Z_{3} \cap Z_{4}$ in $\mathbb{P}^{2 n-5}$. One has an exact sequence

$$
\begin{aligned}
\longrightarrow & H^{2 n-8}\left(Z_{5}\right) \longrightarrow H^{2 n-7}(\bar{Z}) \longrightarrow \\
& H^{2 n-7}\left(Z_{3}\right) \oplus H^{2 n-7}\left(Z_{4}\right) \longrightarrow H^{2 n-7}\left(Z_{5}\right) \longrightarrow .
\end{aligned}
$$

Since $I_{n-1}^{1}=C_{n-1} I_{n-2}^{1}-A_{n-2} I_{n-3}^{1}$ with $C_{n-1}=A_{n-2}, \quad Z_{5}:=Z_{3} \cap Z_{4}=$ $\mathcal{V}\left(I_{n-1}^{1}, A_{n-2}, S_{n-3}\right)=\mathcal{V}\left(A_{n-2}, S_{n-3}\right)$. The defining polynomials of $Z_{5}$ are independent of $B_{1}$ and $B_{2}$, Theorem A $(N=2 n-5, k=2, t=2)$ implies $H_{\text {prim }}^{i}\left(Z_{5}\right)=0$ for $i \leq 2 n-6$. Moreover, $H^{2 n-7}\left(Z_{3}\right) \cong H^{2 n-7}\left(\mathcal{V}\left(A_{n-2}\right)\right)=0$, and the sequence (6.30) yields

$$
H^{2 n-7}(\bar{Z}) \cong H^{2 n-7}\left(Z_{4}\right) \cong H^{2 n-7}\left(\mathcal{V}\left(S_{n-3}, I_{n-1}^{1}\right)\right)
$$

We consider the spectral sequence

$$
E_{1}^{p, q}=\bigoplus_{i_{0}<\ldots<i_{p}} H^{q}\left(\mathcal{V}\left(A_{i_{0}}, \ldots, A_{i_{p}}, I_{n-1}^{1}\right)\right) \Rightarrow H^{p+q}\left(\mathcal{V}\left(S_{n-3}, I_{n-1}^{1}\right)\right)
$$

The only difference to the same situation in the proof of [2, Theorem 11.9] is that we have $C_{n-1}=A_{n-2}$ instead of $B_{n-1}$. Analyzing this sequence similarly, we obtain

$$
H^{2 n-7}\left(Z_{4}\right)=\mathbb{Q}(0)
$$

By (6.29) and (6.31), the sequence (6.27) simplifies to

$$
0 \longrightarrow \mathbb{Q}(0) \longrightarrow H^{2 n-7}\left(Y_{2}\right) \longrightarrow \mathbb{Q}(0) \longrightarrow,
$$

Together with (6.21) and (6.24), this gives us the exact sequence

$$
0 \longrightarrow \mathbb{Q}(-2) \longrightarrow H^{2 n-3}(Y) \longrightarrow \mathbb{Q}(-2) \longrightarrow
$$

Consequently, $H^{2 n-3}(Y) \cong \mathbb{Q}(-2)^{\oplus i}$ for $i=1$ or $i=2$. Collecting (6.14), (6.15) and (6.18) together, we rewrite the sequence (6.17) like

$$
0 \longrightarrow \mathbb{Q}(-1) \longrightarrow H_{\mathrm{prim}}^{2 n-2}(V) \longrightarrow H_{\mathrm{prim}}^{2 n-3}(Y) \longrightarrow
$$


From this, one can describe $H_{\text {prim }}^{2 n-2}(V)$ :

$$
\operatorname{gr}_{2}^{W}\left(H_{\text {prim }}^{2 n-2}(V)\right)=\mathbb{Q}(-1), \quad \operatorname{gr}_{4}^{W}\left(H_{\text {prim }}^{2 n-2}(V)\right)=\mathbb{Q}(-2)^{\oplus j}
$$

and all other $\operatorname{gr}_{W}^{i}$ are zero. Here $0 \leq j \leq i$, thus $j$ equals 0,1 or 2 . Using (6.9), (6.12) and the sequence (6.11) we finally get

$$
\operatorname{gr}_{6}^{W}\left(H_{\text {prim }}^{2 n+2}(X)\right)=\mathbb{Q}(-3) \text { and } \operatorname{gr}_{8}^{W}\left(H_{\text {prim }}^{2 n+2}(X)\right)=\mathbb{Q}(-4)^{\oplus d},
$$

where $d=0,1$ or 2 , all other $\operatorname{gr}_{i}^{W}=0$ and $n \geq 4$. It remains to see what happens when $n=3$. By $(6.25), H_{\text {prim }}^{3}(Y)=0$, thus $H_{\text {prim }}^{4}(V) \cong \mathbb{Q}(-1)$. By (6.9) and (6.11) we now get an exact sequence

$$
0 \longrightarrow H^{8}(X) \longrightarrow Q(-3) \longrightarrow \mathbb{Q}(-4) \longrightarrow .
$$

Applying $\operatorname{gr}_{i}^{W}$, we see that $H^{8}(X) \cong \mathbb{Q}(-3)$.

Remark 6.1. In physics it is known that the periods of $\mathrm{WS}_{3} \times \mathrm{WS}_{3}$ and $\mathrm{WS}_{3} \times \mathrm{WS}_{4}$ are related to $\zeta(3)^{2}$ and $\zeta(3) \zeta(5)$, respectively. For the first case, by duality, we have $H^{9}\left(\mathbb{P}^{9} \backslash X_{\Gamma}\right) \cong Q(-6)$, and the (minus) twist 6 coincides with the weight of $\zeta(3)^{2}$. For the latter, the weight of $\zeta(3) \zeta(5)$ is $3+5=8$, and our computation shows that $H^{11}\left(\mathbb{P}^{11} \backslash X_{\Gamma}\right) \cong Q(-6)$ has the only nontrivial pieces $Q(-8)$ and $Q(-7)^{\oplus d}$. So, in example $\mathrm{WS}_{3} \times \mathrm{WS}_{4}$ we see that exactly the minimal graded pieces of $H^{\text {mid }}\left(X_{\Gamma}\right)$ (or the maximal one of the cohomology of the complement) control the weight of MZV. This is a sort of motivation for studying the minimal graded piece of the cohomology of middle degree for GZZ. And this is also the only piece we can compute.

\section{Acknowledgments}

This article is based on my thesis at the university of Duisburg-Essen. I am very grateful to my advisor Prof. Dr. Hélène Esnault for her constant support and guidance throughout the preparation of the paper. I would also like to thank Dr. Kay Rülling for reading this text and for help.

\section{References}

[1] D. Broadhurst and D. Kreimer, Knots and numbers in $\Phi^{4}$ theory to 7 loops and beyond, Int. J. Mod. Phys. C 6 (1995), 519.

[2] S. Bloch, H. Esnault and D. Kreimer, On motives associated to graph polynomials, Commun. Math. Phys. 267 (2006), 181-225. 
[3] O. Schnetz, Quantum periods: a census of $\phi^{4}$-transcendentals, (arXiv:0801.2856).

[4] F. Brown and K. Yeats, Spanning forest polynomials and the transcendental weight of Feynman graphs, (arXiv:0910.5429).

[5] P. Deligne, Théorie de Hodge, II. Publ. Math. IHES 40 (1974), 5-58.

[6] P. Deligne, Théorie de Hodge, III. Publ. Math. IHES 44 (1974), 5-78.

[7] N.M. Kanz, Review of $\ell$-adic cohomology, Proc. Symp. Pure Math. 55 (1) (1994), 21-30.

[8] P. Deligne, La conjecture de Weil, I. Publ. Math. IHES 43 (1974), 273-307.

UNIVERSITÄT DUISBURG-EsSEN,

FB6, MATHEMATiK,

45117 Essen,

Germany

E-mail address: dzmitry.doryn@uni-due.de

RECEIVED JANUARY 5, 2010 
